\title{
Higgs potential and hidden light Higgs scenario in two Higgs doublet models
}

\author{
Sanghyeon Chang ${ }^{a}$, Sin Kyu Kang ${ }^{b}$, Jong-Phil Lee ${ }^{c}$, and Jeonghyeon Song ${ }^{d}$ \\ ${ }^{a}$ Center for Theoretical Physics of the Universe, IBS, Daejeon 305-811, Korea \\ ${ }^{b}$ School of Liberal Arts, Seoul-Tech, Seoul 139-743, Korea \\ ${ }^{c}$ School of Electrical Engineering and BK21 Plus Humanware Information Technology, \\ Korea University, Seoul 136-713, Korea \\ ${ }^{d}$ School of Physics, Konkuk University, Seoul 143-701, Korea
}

\begin{abstract}
In two Higgs doublet models, there exists an interesting possibility, the hidden light Higgs scenario, that the discovered SM-like Higgs boson is the heavier $C P$-even Higgs boson $H^{0}$ and the lighter $C P$-even $h^{0}$ has not been observed yet in any experiment. We study the current status of this scenario in Types I, II, X, and Y, through the scans of the parameters with all relevant theoretical and experimental constraints. We employ not only the most up-to-date Higgs signal strength measurements with the feed-down effects, but also all the available LHC exclusion limits from heavy Higgs searches. Adjusting the heavier $H^{0}$ to the $125 \mathrm{GeV}$ state while hiding the lighter $h^{0}$ from the LEP Higgs search prohibits the extreme decoupling limit: there exist upper bounds on the masses of the pseudoscalar $A^{0}$ and the charged Higgs $H^{ \pm}$below about $600 \mathrm{GeV}$. In addition, the $Z_{2}$ symmetry is shown to be a good approximate symmetry since the soft $Z_{2}$ symmetry breaking parameter $m_{12}^{2}$ should be less than about $(45 \mathrm{GeV})^{2}$. Most interestingly, a few parameters in the Higgs potential and the related Higgs triple and quartic couplings are shown to be meaningfully constrained by the current data. The double Higgs-strahlung process at an $e^{+} e^{-}$collider is also studied.

PACS numbers: 14.80.Gt, 13.60.Fz, 14.80.Er, 42.62.Hk
\end{abstract}




\section{INTRODUCTION}

The observation of a Higgs boson with mass around $125 \mathrm{GeV}$ at the LHC [1] completes the journey in the standard model (SM): the electroweak symmetry breaking mechanism is uncovered; the mass generation of subatomic particles is most economically explained; the Higgs boson mass, the last unknown parameter in the SM, is precisely measured [2]. The current LHC Higgs data imply that the observed $125 \mathrm{GeV}$ state $h_{125}$ is very similar to the SM Higgs boson [3-7]. Nevertheless there are some vital clues that this is not the end of the road. We do not expect that the ultimate theory of particle physics is the SM which suffers from the gauge hierarchy problem and has no solution to account for $95 \%$ of the energy of the Universe. New physics is inevitable.

Apart from Occam's Razor, there is no reason for prohibiting additional Higgs doublets. Many new physics models contain at least two Higgs doublets, and thus additional Higgs bosons. This extension of the Higgs sector is a good direction toward new physics beyond the SM. The LHC Higgs data on the $125 \mathrm{GeV}$ state may play the role of a compass to show the direction. In addition, both ATLAS and CMS collaborations provide significant exclusion limits from the null results in the searches for the heavy neutral and charged Higgs bosons. In the ordinary setup where the observed is the lightest $C P$-even neutral Higgs boson $h^{0}$, the compass naturally points to the decoupling limit [8] where the other Higgs states are very heavy. The phenomenology of the decoupling limit generically mimics that of the SM. Even if the current experimental status, the SM-like $125 \mathrm{GeV}$ state without any signal of other Higgs bosons, might keep in the future, the verification or invalidation of a specific new physics model will be postponed till the next generation collider.

If the observed $125 \mathrm{GeV}$ state is a heavier $C P$-even neutral Higgs boson $H^{0}$, however, the LHC Higgs data play a much more significant role in characterizing a specific model. Adjusting the heavier $H^{0}$ to $h_{125}$ as well as hiding the lighter $h^{0}$ from low energy experiment data constrain the new physics model strongly. We call this possibility the hidden light Higgs scenario. If the current LHC data can specify the Higgs potential in this scenario thanks to the expected strong constraints, it will give important implications on the dynamics of the electroweak phase transition [9, 10], and the measurement of the cubic and quartic self-couplings of Higgs bosons in the future collider [11, 12].

As the simplest extension of the SM Higgs sector, we consider a two Higgs doublet model 
TABLE I: Summary of constraints.

\begin{tabular}{|c|c|}
\hline $\begin{array}{l}\text { Theoretical stability } \\
\text { (yellow) }\end{array}$ & $\begin{array}{l}\cdot V_{H} \text { to be bounded below } \\
\cdot \text { Unitarity } \\
\text { - Perturbativity of quartic couplings }\end{array}$ \\
\hline $\begin{array}{l}\text { Pre-LHC bounds } \\
\text { (green) }\end{array}$ & $\begin{array}{l}\cdot \text { LEP bounds on } h^{0} \text { and } H^{ \pm} \\
\cdot \Delta \rho \text { in the electroweak precision data } \\
\cdot \text { FCNC like } \Delta M_{B_{d}} \text { and } b \rightarrow s \gamma \\
\cdot \text { Top quark decay into } H^{ \pm}\end{array}$ \\
\hline $\begin{array}{l}\text { LHC bounds } \\
\text { (red) }\end{array}$ & $\begin{array}{l}\cdot m_{H}=125 \mathrm{GeV} \\
\cdot \text { LHC search for } H^{ \pm} \text {via } p p \rightarrow t \bar{t} H^{ \pm} \text {followed by } H^{ \pm} \rightarrow \tau \nu \\
\cdot \text { LHC search for } A^{0} \text { via } g g \rightarrow A^{0} \rightarrow \gamma \gamma, \tau^{+} \tau^{-} \text {and } b \bar{b} \rightarrow A^{0} \rightarrow \tau^{+} \tau^{-} \\
\text {- Global } \chi^{2} \text { fit to the LHC Higgs data including } \\
\text { (i) Additional decay channels of } H^{0} \rightarrow h^{0} h^{0}, A^{0} A^{0}, H^{+} H^{-}, W^{ \pm} H^{\mp} \\
\text { (ii) the "feed-down" }\end{array}$ \\
\hline
\end{tabular}

(2HDM) [13] with $C P$ invariance and softly broken $Z_{2}$ symmetry [14]. There exist five physical Higgs bosons, the light $C P$-even scalar $h^{0}$, the heavy $C P$-even scalar $H^{0}$, the $C P$ odd pseudoscalar $A^{0}$, and two charged Higgs bosons $H^{ \pm}$. The general Higgs potential has 7 parameters. According to the $Z_{2}$ charges of the SM quarks and leptons, there exist four types of 2HDM: Type I, Type II, Type X, and Type Y [15, 16]. In the normal setup of $h^{0}=h_{125}$, there are extensive studies on the global fit analysis of the Higgs signal strengths as well as the phenomenology of the other heavy Higgs bosons [17 28]. The hidden light Higgs scenario is also naturally accommodated in the 2HDM [6, 29 2 ]5: $H^{0}$ is the $125 \mathrm{GeV}$ state and $h^{0}$ has not been observed yet.

A comprehensive study of the current status of the 2HDM Type I and Type II by including the heavy Higgs search data was first performed in Ref. [36]. Similar comprehensive studies in other setup such as very light Higgs bosons [31] or the minimal supersymmetric standard model [37] were followed up. We extend the study, focusing on the question of how much the current data constrain the Higgs potential in the hidden light Higgs scenario. To answer the question, we consider more extended constraints than in Ref. [36], particularly those from 
the LHC heavy Higgs searches. We classify the theoretical and phenomenological constraints into three categories: theoretical bounds, pre-LHC bounds, and LHC bounds.

The details of each step are summarized in Table [. The theoretical bounds demand the boundedness of the Higgs potential [38], unitarity [39, 40, and perturbativity. The "preLHC" bounds include the LEP bounds on $h^{0}$ [41, 42] and $H^{ \pm}$[43], $\Delta \rho$ in the electroweak precision data [44, 45], the flavor changing neutral current (FCNC) data such as $\Delta M_{B_{d}}$ and $b \rightarrow s \gamma$ [46, 47], and the top quark decay of $t \rightarrow H^{+} b$ [48. The "LHC" bounds are based not only on the Higgs signal strength measurements from LHC8, but also on the exclusion limits from all the heavy Higgs searches including $H^{ \pm} \rightarrow \tau^{ \pm} \nu_{\tau}$ [49, 50], $H^{+} \rightarrow c \bar{s}$ [51], $g g \rightarrow A^{0} \rightarrow \gamma \gamma$ [52, 53], $g g \rightarrow A^{0} \rightarrow \tau^{+} \tau^{-}$[54, 55], and $b \bar{b} \rightarrow A^{0} \rightarrow \tau^{+} \tau^{-}$[54, [55]. For the $125 \mathrm{GeV}$ state data, we perform the comprehensive global $\chi^{2}$ analysis by including $H^{0} \rightarrow h^{0} h^{0}$ and $H^{0} \rightarrow A^{0} A^{0}$ as well as "feed-down" (FD) contributions from the production of heavier Higgs bosons through their decay into $H^{0}$ [36, 56], not by just limiting the FD signal strength value.

We shall show that in all four types, the hidden light Higgs scenario is consistent with the data, as good as the SM. In addition, the survived parameter points have many interesting implications. Major ones are as follows: (i) there exist upper bounds on the heavy Higgs bosons like $m_{A, H^{ \pm}} \lesssim 600 \mathrm{GeV}$; (ii) the soft $Z_{2}$ symmetry breaking term $m_{12}^{2}$ is strongly constrained to be below about $(45 \mathrm{GeV})^{2}$ in most cases; (iii) in Type I and $\mathrm{X}$, a light $m_{A}$ (as low as $20 \mathrm{GeV}$ ) and $m_{H^{ \pm}}$(as low as $100 \mathrm{GeV}$ ) are allowed; (iv) the triple Higgs coupling $g_{H H H}$ is very like the SM Higgs triple coupling, and $g_{h H H}$ is similar to the SM value; (v) the quartic Higgs couplings $g_{H H H H}$ and $g_{h H H H}$ are similar to the SM value. Inspired by the almost fixed $g_{H H H}$ and $g_{h H H}$, we study the double Higgs-strahlung at an $e^{+} e^{-}$collider, $e^{+} e^{-} \rightarrow Z^{0} H^{0} H^{0}$, which can be highly enhanced in some parameter space where the resonance production of $A^{0}$ is allowed. These are our main results.

The paper is organized as follows. In Sec. II] we briefly review the 2HDM. Section III] summarizes the constraints. Section IV presents our results, the allowed parameter space stage by stage. In Sec. V], we show the Higgs triple and quartic couplings in the allowed parameter space, and study the future prospect of $e^{+} e^{-} \rightarrow Z^{0} H^{0} H^{0}$. Section VI contains our conclusions. 


\section{BRIEF REVIEW OF THE 2HDM}

A 2HDM [13] introduces two complex $S U(2)_{L}$ Higgs doublet scalar fields, $\Phi_{1}$ and $\Phi_{2}$. Both $\Phi_{1}$ and $\Phi_{2}$ develop nonzero vacuum expectation values as $\left\langle\Phi_{1,2}\right\rangle=\left(0, v_{1,2} / \sqrt{2}\right)^{T}$, which generate the electroweak symmetry breaking. When parametrising $t_{\beta}=v_{2} / v_{1}$, one linear combination $H_{1}=c_{\beta} \Phi_{1}+s_{\beta} \Phi_{2}$ has nonzero vacuum expectation value of $v=\sqrt{v_{1}^{2}+v_{2}^{2}}=246 \mathrm{GeV}$, while its orthogonal combination $H_{2}=-s_{\beta} \Phi_{1}+c_{\beta} \Phi_{2}$ acquires zero vacuum expectation value. For simplicity of notation, we take $s_{x}=\sin x, c_{x}=\cos x$, and $t_{x}=\tan x$. We define the fluctuation fields about the minima $v_{1}$ and $v_{2}$ as

$$
\Phi_{i}=\left(\begin{array}{c}
\phi_{i}^{+} \\
\frac{v_{i}+\rho_{i}+i \eta_{i}}{\sqrt{2}}
\end{array}\right), \quad i=1,2 .
$$

In order to avoid FCNC at the lowest order, a discrete $Z_{2}$ symmetry is imposed, under which $\Phi_{1} \rightarrow \Phi_{1}$ and $\Phi_{2} \rightarrow-\Phi_{2}[14]$.

The most general potential with $C P$ invariance and softly broken $Z_{2}$ symmetry is

$$
\begin{aligned}
V= & m_{11}^{2} \Phi_{1}^{\dagger} \Phi_{1}+m_{22}^{2} \Phi_{2}^{\dagger} \Phi_{2}-m_{12}^{2}\left(\Phi_{1}^{\dagger} \Phi_{2}+\text { H.c. }\right) \\
& +\frac{1}{2} \lambda_{1}\left(\Phi_{1}^{\dagger} \Phi_{1}\right)^{2}+\frac{1}{2} \lambda_{2}\left(\Phi_{2}^{\dagger} \Phi_{2}\right)^{2}+\lambda_{3}\left(\Phi_{1}^{\dagger} \Phi_{1}\right)\left(\Phi_{2}^{\dagger} \Phi_{2}\right)+\lambda_{4}\left(\Phi_{1}^{\dagger} \Phi_{2}\right)\left(\Phi_{2}^{\dagger} \Phi_{1}\right) \\
& +\frac{1}{2} \lambda_{5}\left[\left(\Phi_{1}^{\dagger} \Phi_{2}\right)^{2}+\text { H.c. }\right] .
\end{aligned}
$$

The $C P$ invariance requires all of the parameters to be real, and $m_{12}^{2}$ breaks the $Z_{2}$ symmetry softly. Note that the $m_{12}^{2}$ parameter can be negative. Using the tadpole condition, $m_{11}$ and $m_{22}$ can be written in terms of $v, t_{\beta}$, and $\lambda_{1, \cdots, 5}$. The Higgs potential has 7 free parameters of $m_{12}^{2}, t_{\beta}$, and $\lambda_{1, \cdots, 5}$.

The charged Higgs boson $H^{ \pm}$is a linear combination of $\phi_{1}^{ \pm}$and $\phi_{2}^{ \pm}$, and the pseudoscalar $A^{0}$ is a linear combination of $\eta_{1}$ and $\eta_{2}$. Their orthogonal states are Goldstone modes $G^{ \pm}$ and $G^{0}$, respectively. And their masses are

$$
m_{H^{ \pm}}^{2}=\frac{m_{12}^{2}}{c_{\beta} s_{\beta}}-\left(\lambda_{4}+\lambda_{5}\right) v^{2}, \quad m_{A}^{2}=\frac{m_{12}^{2}}{c_{\beta} s_{\beta}}-2 \lambda_{5} v^{2}
$$

The physical $C P$-even Higgs bosons $h^{0}$ and $H^{0}$ are obtained through the diagonalization of the mass squared matrix $\mathcal{M}_{0}^{2}$ with the mixing angle $\alpha$, given by

$$
\mathcal{M}_{0}^{2}=\left(\begin{array}{ll}
\mathcal{M}_{11}^{2} & \mathcal{M}_{12}^{2} \\
\mathcal{M}_{12}^{2} & \mathcal{M}_{22}^{2}
\end{array}\right)
$$


where

$$
\begin{aligned}
& \mathcal{M}_{11}^{2}=m_{12}^{2} t_{\beta}^{2}+\frac{\lambda_{1} v^{2}}{1+t_{\beta}^{2}}, \quad \mathcal{M}_{22}^{2}=\frac{m_{12}^{2}}{t_{\beta}^{2}}+\lambda_{2} v^{2} \frac{t_{\beta}^{2}}{1+t_{\beta}^{2}} \\
& \mathcal{M}_{12}^{2}=-m_{12}^{2}+\lambda_{345} v^{2} \frac{t_{\beta}}{1+t_{\beta}^{2}},
\end{aligned}
$$

where $\lambda_{345}=\lambda_{3}+\lambda_{4}+\lambda_{5}$. The masses of neutral Higgs $m_{h}$ and $m_{H}$ are

$$
m_{H, h}^{2}=\frac{1}{2}\left[\mathcal{M}_{11}^{2}+\mathcal{M}_{22}^{2} \pm \sqrt{\left(\mathcal{M}_{11}^{2}-\mathcal{M}_{22}^{2}\right)^{2}+4\left(\mathcal{M}_{12}^{2}\right)^{2}}\right] .
$$

The SM Higgs field, which corresponds to $H_{1}$, becomes

$$
h_{\mathrm{SM}}=s_{\beta-\alpha} h^{0}+c_{\beta-\alpha} H^{0} .
$$

If $c_{\beta-\alpha}=1$ and $m_{H}=125 \mathrm{GeV}, H^{0}$ has the same properties as the SM Higgs boson. This is called the alignment limit [57]:

$$
\text { The alignment limit for } H^{0}=h_{\mathrm{SM}}: \quad c_{\beta-\alpha}=1 \text {. }
$$

As shall be shown, the allowed parameters by all the constraints are distributed around the alignment limit. The alignment limit maximizes or minimizes some triple couplings of Higgs bosons with weak gauge bosons or other Higgs bosons. We classify them into two categories, one proportional to $s_{\beta-\alpha}$ and the other proportional to $c_{\beta-\alpha}$ :

$$
\begin{aligned}
& s_{\beta-\alpha}: g_{h W^{+} W^{-}}, \quad g_{h Z Z}, \quad g_{Z A H}, \quad g_{W^{ \pm} H^{\mp} H}, \\
& c_{\beta-\alpha}: g_{H W^{+} W^{-}}, \quad g_{H Z Z}, \quad g_{Z A h}, \quad g_{W^{ \pm} H^{\mp} h}, \quad g_{H h h} .
\end{aligned}
$$

In the hidden light Higgs scenario, the couplings proportional to $s_{\beta-\alpha}$ vanish in the alignment limit.

Yukawa couplings of Higgs bosons are different according to the 2HDM type. Focusing on the $125 \mathrm{GeV}$ state $H^{0}$, we present the normalized Yukawa couplings by the SM values, $\hat{y}_{u u, d d, \ell \ell}^{H}$, in terms of $c_{\beta-\alpha}$ and $s_{\beta-\alpha}$ :

\begin{tabular}{c|cc} 
& $c_{\beta-\alpha}-\frac{s_{\beta-\alpha}}{t_{\beta}}$ & $c_{\beta-\alpha}+t_{\beta} s_{\beta-\alpha}$ \\
\hline Type I & $\hat{y}_{u u}^{H}, \hat{y}_{d d}^{H}, \hat{y}_{\ell \ell}^{H}$ & \\
Type II & $\hat{y}_{u u}^{H}$ & $\hat{y}_{d d}^{H}, \hat{y}_{\ell \ell}^{H}$ \\
Type X & $\hat{y}_{u u}^{H}, \hat{y}_{d d}^{H}$ & $\hat{y}_{\ell \ell}^{H}$ \\
Type Y & $\hat{y}_{u u}^{H}, \hat{y}_{\ell \ell}^{H}$ & $\hat{y}_{d d}^{H}$
\end{tabular}


Note that in the exact alignment limit $\left(c_{\beta-\alpha}=1\right)$, all of the Yukawa couplings for $H^{0}$ are the same as in the SM. There are two kinds of deviation from the alignment limit: one is proportional to $t_{\beta}$, and the other to $1 / t_{\beta}$. Since FCNC constrains $t_{\beta} \gtrsim 1$, those proportional to $t_{\beta} s_{\beta-\alpha}$ yield much larger deviation from the SM Yukawa coupling. As shown in Eq. (10), Type I has common Yukawa couplings, which have the $t_{\beta}$-suppressed deviation from the alignment. On the while, Type II has the $t_{\beta}$-enhanced deviation for the down-type quark and charged lepton Yukawa couplings. As shall be shown later, Type II is most strongly constrained by the current LHC Higgs data.

\section{CONSTRAINTS}

We constrain the hidden light Higgs scenario in the 2HDM by sequentially taking three steps. The first step (yellow) is to apply theoretical conditions, the second (green) is to use all reliable experimental constraints before the LHC data, and the last step (red) is to include the LHC Higgs data that consist of the observation of the $125 \mathrm{GeV}$ state as well as the exclusion limits from the searches for the other Higgs bosons heavier than 125 $\mathrm{GeV}$. In what follows, each coloured point (yellow, green, or red) represents the surviving parameters at $95 \%$ C.L., up to the corresponding step. For example, the green points satisfy the theoretical and pre-LHC bounds. We summarise the constraints in Table I.

\section{A. Theoretical constraints}

(i) The Higgs potential to be bounded from below: As proven in Ref. [38], the scalar potential in Eq. (2) is bounded from below if and only if the following conditions are satisfied:

$$
\lambda_{1}>0, \quad \lambda_{2}>0, \quad \lambda_{3}>-\sqrt{\lambda_{1} \lambda_{2}}, \quad \lambda_{3}+\lambda_{4}-\left|\lambda_{5}\right|>-\sqrt{\lambda_{1} \lambda_{2}} .
$$

(ii) Unitarity: Tree level perturbative unitarity requires for the absolute values of the 
followings to be less than $8 \pi$ [39, 40]:

$$
\begin{aligned}
a_{ \pm} & =\frac{3}{2}\left(\lambda_{1}+\lambda_{2}\right) \pm \sqrt{\frac{9}{4}\left(\lambda_{1}-\lambda_{2}\right)^{2}+\left(2 \lambda_{3}+\lambda_{4}\right)^{2}} \\
b_{ \pm} & =\frac{1}{2}\left(\lambda_{1}+\lambda_{2} \pm \sqrt{\left(\lambda_{1}-\lambda_{2}\right)^{2}+4 \lambda_{4}^{2}}\right) \\
c_{ \pm} & =\frac{1}{2}\left(\lambda_{1}+\lambda_{2} \pm \sqrt{\left(\lambda_{1}-\lambda_{2}\right)^{2}+4 \lambda_{5}^{2}}\right), \\
f_{+} & =\lambda_{3}+2 \lambda_{4}+3 \lambda_{5}, \quad f_{-}=\lambda_{3}+\lambda_{5}, \quad f_{1}=\lambda_{3}+\lambda_{4}, \\
e_{1} & =\lambda_{3}+2 \lambda_{4}-3 \lambda_{5}, \quad e_{2}=\lambda_{3}-\lambda_{5}, \quad p_{1}=\lambda_{3}-\lambda_{4} .
\end{aligned}
$$

(iii) Perturbativity: We first demand the bare quartic couplings in the Higgs potential to satisfy the perturbativity as

$$
\left|\lambda_{i}\right|<4 \pi, \quad i=1, \cdots, 5
$$

In addition, the magnitudes of the quartic couplings among physical Higgs states like $g_{\varphi_{i} \varphi_{j} \varphi_{k} \varphi_{\ell}}\left(\phi_{i}=h^{0}, H^{0}, A^{0}, H^{ \pm}\right)$are required to be smaller than $4 \pi$.

Here we do not require that the $2 \mathrm{HDM}$ vacuum should be the global minimum of the potential [58], because the existence of a false vacuum (local minimum) is acceptable if its lifetime is longer than the age of the Universe. Unquestionably if the lifetime of a false vacuum is shorter, the corresponding parameter space should be excluded. Since the calculation of the $2 \mathrm{HDM}$ vacuum lifetime is beyond the scope of this study, we take a conservative stance to ignore the global minimum condition.

\section{B. pre-LHC bounds}

(i) The LEP bounds on $h^{0}$ and $H^{ \pm}$: One of the most direct channels to probe a light Higgs boson with mass below $120 \mathrm{GeV}$ is the Higgs-strahlung at the LEP. We use the strongest upper bound on the event rate of $e^{+} e^{-} \rightarrow Z^{0} h^{0} \rightarrow Z^{0} j j$ [41, 42]. Another important result from the LEP is the direct production limit on the charged Higgs boson mass as [43]:

$$
m_{H^{ \pm}} \geq 80 \mathrm{GeV}
$$

(ii) $\Delta \rho$ in the electroweak precision data: The $\Delta \rho$ parameter from the electroweak precision measurement has additional contributions in the 2HDM through the heavy neutral Higgs bosons $\left(A^{0}\right.$ in the hidden light Higgs scenario) as well as the charged Higgs bosons [44, 45]. 
With the observed Higgs boson mass, its global fit result has been improved significantly as 43

$$
\Delta \rho=0.00040 \pm 0.00024
$$

It is known that the new contribution is suppressed if $m_{A} \simeq m_{H^{ \pm}}$or $m_{H} \simeq m_{H^{ \pm}}$[59, 60]. As shall be discussed, the $m_{H} \simeq m_{H^{ \pm}} \simeq 125 \mathrm{GeV}$ case is prohibited by the FCNC constraints in Types II and Y.

(iii) FCNC processes such as $\Delta M_{B_{d}}$ and $b \rightarrow s \gamma$ : In the $2 \mathrm{HDM}$, the charged Higgs boson contributes to various FCNC processes through the loop. We consider two sensitive FCNC processes, $b \rightarrow s \gamma$ [46, 47] and $\Delta M_{B_{d}}$ [47, 61]. $\Delta M_{B_{d}}$ excludes small $t_{\beta}$ region for all types, while $b \rightarrow s \gamma$ further excludes the light charged Higgs mass region in Type II and Type Y. Other processes such as $\varepsilon_{K}$ [62] and $R_{b}$ [63, 64] impose weaker constraints [65]. We do not consider the measurements of $R\left(D^{(*)}\right) \equiv \operatorname{Br}\left(\bar{B} \rightarrow D^{(*)} \tau^{-} \bar{\nu}_{\tau}\right) / \operatorname{Br}\left(\bar{B} \rightarrow D^{(*)} l^{-} \bar{\nu}_{l}\right)$ from BaBar [66 $]^{1}$.

(iv) Bound from $t \rightarrow b H^{+}$: A light charged Higgs boson could have appeared in the top decay into $b H^{+}$if kinematically allowed. We include the Tevatron search results of the upper bounds on $\operatorname{Br}\left(t \rightarrow b H^{+}\right)$[48].

\section{LHC bounds}

(i) Higgs mass bounds: Both ATLAS and CMS collaborations measured the Higgs boson mass with high precision [71, 72]. The combined result is [73]

$$
m_{H}=125.09 \pm 0.21 \text { (stat) } \pm 0.11 \text { (syst) } \mathrm{GeV} .
$$

We demand that $m_{H}$ be within $2 \sigma$. In addition, we exclude the degenerate cases of $m_{H}=m_{A}$ and $m_{h}=m_{H}$ in order to avoid the possible contributions from $h^{0}$ or $A^{0}$ to the observed Higgs signal strengths. Both $m_{h}$ and $m_{A}$ should lie outside $m_{H}$ at $2 \sigma$.

(ii) LHC search for the charged Higgs boson: The search strategy of the charged Higgs boson at the LHC is different according to its mass. For $m_{H^{ \pm}}>m_{t}+m_{b}$, the main decay mode is into $t \bar{b}[74]$. For lighter charged Higgs boson than the top quark, two decay channels

1 Recently, LHCb 67] reported $2.1 \sigma$ excess of $R\left(D^{*}\right)$ over the SM predictions, and Belle 68] presented a new measurements of $R(D)$ and $R\left(D^{*}\right)$ which are not significantly deviated from both the SM prediction and the measured values at BaBar [66] and $\mathrm{LHCb}$. 
TABLE II: Summary of the LHC Higgs signal strengths at 7 and $8 \mathrm{TeV}$.

\begin{tabular}{|c|c|c|}
\hline Production & ATLAS & CMS \\
\hline$g g \mathrm{~F}+t \bar{t} h$ & $\begin{array}{l}\widetilde{R}_{\gamma \gamma}^{g g \mathrm{~F}}=1.32 \pm 0.38[3], \widetilde{R}_{\gamma \gamma}^{t \bar{t} h}=1.3_{-1.7}^{+2.6}[4] \\
\widetilde{R}_{W W}^{g g \mathrm{~F}}=1.01_{-0.20}^{+0.27}[79] \\
\widetilde{R}_{Z Z}^{g g \mathrm{~F}+t \bar{t} h}=1.52_{-0.65}^{+0.85}[81] \\
\widetilde{R}_{\tau \tau}^{g g \mathrm{~F}}=1.93_{-1.15}^{+1.45}[83] \\
\widetilde{R}_{b \bar{t}}^{t \bar{t} h}=1.7 \pm 1.4[85,86]\end{array}$ & $\begin{array}{l}\widetilde{R}_{\gamma \gamma}^{g g \mathrm{~F}+t \bar{t} h}=1.13_{-0.31}^{+0.37}[5] \\
\widetilde{R}_{W W}^{g g \mathrm{~F}}=0.74_{-0.20}^{+0.22}[80] \\
\widetilde{R}_{Z Z}^{g g \mathrm{~F}+t \bar{t} h}=0.80_{-0.36}^{+0.46}[82] \\
\widetilde{R}_{\tau \tau}^{g g \mathrm{~F}}=0.93 \pm 0.42[84 \\
\widetilde{R}_{b \bar{b}}^{t \bar{t} h}=0.67_{-1.33}^{+1.35}[87\end{array}$ \\
\hline $\mathrm{VBF}+V h$ & $\begin{array}{l}\widetilde{R}_{\gamma \gamma}^{\mathrm{VBF}}=0.8 \pm 0.7, \\
\widetilde{R}_{\gamma \gamma}^{W H}=1.0 \pm 1.6, \widetilde{R}_{\gamma \gamma}^{Z H}=0.1_{-0.1}^{+3.7}[3] \\
\widetilde{R}_{W W}^{\mathrm{VBF}}=1.28_{-0.45}^{+0.53}[79] \\
\widetilde{R}_{Z Z}^{\mathrm{VBF}+V h}=0.90_{-2.0}^{+4.5}[81] \\
\widetilde{R}_{\tau \tau}^{\mathrm{VBF}+V h}=1.24_{-0.54}^{+0.58}[83] \\
\widetilde{R}_{b \bar{b}}^{V h}=0.51_{-0.37}^{+0.40}[88\end{array}$ & $\begin{array}{l}\widetilde{R}_{\gamma \gamma}^{\mathrm{VBF}+V h}=1.15_{-0.58}^{+0.63}[5] \\
\widetilde{R}_{W W}^{\mathrm{VBF}}=0.60_{-0.46}^{+0.57}, \widetilde{R}_{W W}^{V h}=0.39_{-1.87}^{+1.97}[80] \\
\widetilde{R}_{Z Z}^{\mathrm{VBF}+V h}=1.7_{-2.1}^{+2.2}[82] \\
\widetilde{R}_{\tau \tau}^{\mathrm{VBF}}=0.94 \pm 0.41, \widetilde{R}_{\tau \tau}^{V h}=-0.33 \pm 1.02[84] \\
\widetilde{R}_{b \bar{b}}^{\mathrm{VBF}}=0.7 \pm 1.4\left[87, \quad \widetilde{R}_{b \bar{b}}^{V h}=1.0 \pm 0.5[89\right.\end{array}$ \\
\hline
\end{tabular}

are searched, $H^{ \pm} \rightarrow \tau^{ \pm} \nu_{\tau}$ [49, 50] and $H^{+} \rightarrow c \bar{s}$ [51]. Since the direct production of the charged Higgs boson is very small, the bound for the charged Higgs boson is weak in general. The strongest bound is from $p p \rightarrow t \bar{t} \rightarrow b \bar{b} H^{ \pm} W^{\mp}$, followed by $H^{ \pm} \rightarrow \tau^{ \pm} \nu$. We include the upper bounds on $\operatorname{Br}\left(t \rightarrow H^{+} b\right) \times \operatorname{Br}\left(H^{+} \rightarrow \tau^{+} \nu_{\tau}\right)$.

(iii) $L H C$ search for $A^{0}$ : In the hidden light Higgs scenario, only $A^{0}$ is the heavy neutral Higgs boson. Up to now, there are no significant excesses in the heavy neutral Higgs search, which provides the exclusion limit. We include the $g g \rightarrow A^{0} \rightarrow \gamma \gamma$ [52, 53], $g g \rightarrow A^{0} \rightarrow$ $\tau^{+} \tau^{-}$[54, 55], and $b \bar{b} \rightarrow A^{0} \rightarrow \tau^{+} \tau^{-}$[54, 55]. Note that the $W^{+} W^{-}$and $Z^{0} Z^{0}$ decay channels are not relevant for the pseudoscalar $A^{0}$. Another important decay channel is into $t \bar{t}$, which is dominant if $m_{A}>2 m_{t}$ and $t_{\beta} \lesssim 10$ [60]. Although both ATLAS and CMS collaborations reported the $t \bar{t}$ resonance search results [75], the interpretation is very challenging at a hadron collider because the interference with the QCD continuum background causes various shapes in the $t \bar{t}$ invariant mass distribution [76 78 . Since the interference effects have not been included yet in the experiment analysis, we do not consider this $t \bar{t}$ channel.

(iv) the global fit to the LHC 125 GeV state data with the FD effects: The discovery of the Higgs boson is not based on a single observation of a resonance, but more than 200 channels. Any new physics model should explain the whole LHC Higgs data, which is commonly 
analysed through the global $\chi^{2}$ fit. We parameterize each signal rate by $R_{\text {decay }}^{\text {prodion }}$, the ratio of the observed event rate to the SM expectation in the specific channel, and identify it with the signal strength modifier $\mu \equiv \sigma / \sigma_{\mathrm{SM}}$. In the $2 \mathrm{HDM}, R$ 's are not generally equal to one as in the SM. The latest experimental values, denoted by $\tilde{R}$ 's, are summarised in Table II. We perform global $\chi^{2}$ fits of 7 model parameters to the observed Higgs signal strength $\widetilde{R}_{i}$

In the $2 \mathrm{HDM}$, there are three sources to deviate the signal strength value from one. First, the effective couplings of $H^{0}$ with the SM particles can be different from those in the SM, which happens when $c_{\beta-\alpha} \neq 1$. Second, there are additional decay channels of $H^{0}$ into $h^{0} h^{0}$, $A^{0} A^{0}, Z^{0} A^{0}, W^{ \pm} H^{\mp}$, and $H^{+} H^{-}$. Too large decay rates of new decay modes enhance the total decay rate of $H^{0}$, which affects the observed signal strengths. We include these decay rates to the global $\chi^{2}$ fit. In the hidden light Higgs scenario, the $H^{0} \rightarrow h^{0} h^{0}$ mode excludes considerable parameter space even in the alignment limit, since the vertex is proportional to $c_{\beta-\alpha}$. On the contrary, the vertices of $Z^{0}-A^{0}-H^{0}$ and $W^{ \pm}-H^{\mp}-H^{0}$ are proportional to $s_{\beta-\alpha}$, and thus $H^{0} \rightarrow Z^{0} A^{0}, W^{ \pm} H^{\mp}$ are suppressed in the alignment limit: see Eq. (9). The LEP bounds of $m_{H^{ \pm}} \geq 80 \mathrm{GeV}$ kinematically suppresses $H^{0} \rightarrow H^{+} H^{-}$.

The third source for the deviation is the FD effects [36, 56]: the inclusive decay of heavy Higgs states into $H^{0}$ yields more events in the $125 \mathrm{GeV}$ state, which renders the $H^{0}$ not to be SM-like even in the alignment limit. In the hidden light Higgs scenario, dominant contribution to the FD effects is from the inclusive decay of $A^{0}$ into $H^{0}$. The $H^{ \pm}$contribution is negligible since its production at the LHC is too small. The general probability of the inclusive production of $H^{0}$ from $A^{0}$ decay is [36]

$$
\begin{aligned}
\mathcal{P}_{\mathrm{FD}}\left(A^{0} \rightarrow H^{0}+X\right)= & 2 \operatorname{Br}\left(A^{0} \rightarrow H^{+} H^{-}\right) \operatorname{Br}\left(H^{+} \rightarrow W^{+} H^{0}\right)^{2}+\operatorname{Br}\left(A^{0} \rightarrow Z^{0} H^{0}\right) \\
& +2 \operatorname{Br}\left(A^{0} \rightarrow W^{-} H^{+}\right) \operatorname{Br}\left(H^{+} \rightarrow W^{+} H^{0}\right) \\
& +2 \operatorname{Br}\left(A^{0} \rightarrow H^{+} H^{-}\right) \operatorname{Br}\left(H^{+} \rightarrow W^{+} H^{0}\right)\left\{1-\operatorname{Br}\left(H^{+} \rightarrow W^{+} H^{0}\right)\right\} .
\end{aligned}
$$

As shall be shown in the next section, the "pre-LHC" constraints allow two kinds of regions in the $\left(m_{H^{ \pm}}, m_{A}\right)$ parameter space. One is $m_{H^{ \pm}} \simeq m_{A}$ in all four types, and the other allows $m_{H^{ \pm}} \approx 100 \mathrm{GeV}$. Only the $\operatorname{Br}\left(A^{0} \rightarrow Z^{0} H^{0}\right)$ in Eq. (17) is kinematically relevant.

We define new FD signal strengths as

$$
\mu_{i i}^{\mathrm{FD}: \mathrm{ZH}}=\frac{\sigma\left(p p \rightarrow g g \rightarrow A^{0}\right) \operatorname{Br}\left(A^{0} \rightarrow Z^{0} H^{0}\right)}{\sigma\left(p p \rightarrow Z^{0} h_{\mathrm{SM}}\right)} \times \frac{\operatorname{Br}\left(H^{0} \rightarrow i i\right)}{\operatorname{Br}\left(h_{\mathrm{SM}} \rightarrow i i\right)},
$$


where $i=\gamma, W, Z, \tau, b^{2}$. We add $\mu_{i i}^{\mathrm{FD}: Z H}$ to $R_{i i}^{Z H}$ and perform the global $\chi^{2}$ fit to the observed $\widetilde{R}$ 's.

\section{RESULTS}

For the 7 parameters of $\lambda_{1, \cdots, 5}, t_{\beta}$, and $m_{12}^{2}$, we randomly generate $2 \times 10^{10}$ points to scan over the following ranges:

$$
\begin{aligned}
& \lambda_{1,2} \in[0,4 \pi], \quad \lambda_{3,4,5} \in[-4 \pi, 4 \pi], \\
& t_{\beta} \in[1,50], \quad m_{12}^{2} \in\left[-(2 \mathrm{TeV})^{2},(2 \mathrm{TeV})^{2}\right] .
\end{aligned}
$$

We apply three steps of bounds:

Step-1 (yellow) theoretical bounds;

Step-2 (green) the pre-LHC bounds;

Step-3 (red) the LHC bounds.

The detailed conditions are summarised in Table I. In what follows, yellow, green, and red points denote the survived parameters after Step-1, Step-2, and Step-3 bounds, respectively. Note that the bound conditions are accumulatively applied. The red points satisfy all the bounds.

(i) High reliability of the hidden light Higgs scenario: We find that the hidden light Higgs scenario is consistent with all the current data. Out of $2 \times 10^{10}$ parameter sets and at $95 \%$ C.L., $2.2 \times 10^{4}$ points survived in Type I, $0.74 \times 10^{4}$ in Type II, $1.1 \times 10^{4}$ in Type X, and $1.4 \times 10^{4}$ in Type Y. Limited but substantial parameter space is consistent with the current data. Type I has the largest allowed parameter space while Type II has the smallest. The minimum values of $\chi^{2}$ per degree of freedom are $0.40,0.51,0.51,0.50$ in Type I, II, X, and $\mathrm{Y}$, respectively. In the $\mathrm{SM}$, it is 0.49 . The best fit points in the $2 \mathrm{HDM}$ explain the current data at least as good as the SM.

(ii) Upper bounds on $m_{A}$ and $m_{H^{ \pm}}$: A unique feature of the hidden light Higgs scenario is the presence of the upper bounds on heavy Higgs boson masses, $m_{A}$ and $m_{H^{ \pm}}$. In the

\footnotetext{
${ }^{2}$ Our definition has additional factor of $\operatorname{Br}\left(H^{0} \rightarrow i i\right) / \operatorname{Br}\left(h_{\mathrm{SM}} \rightarrow i i\right)$ compared with the $\mu^{\mathrm{FD}}$ in Ref. 36], which is the ratio of the FD production to direct production in the $2 \mathrm{HDM}$.
} 
Type-I

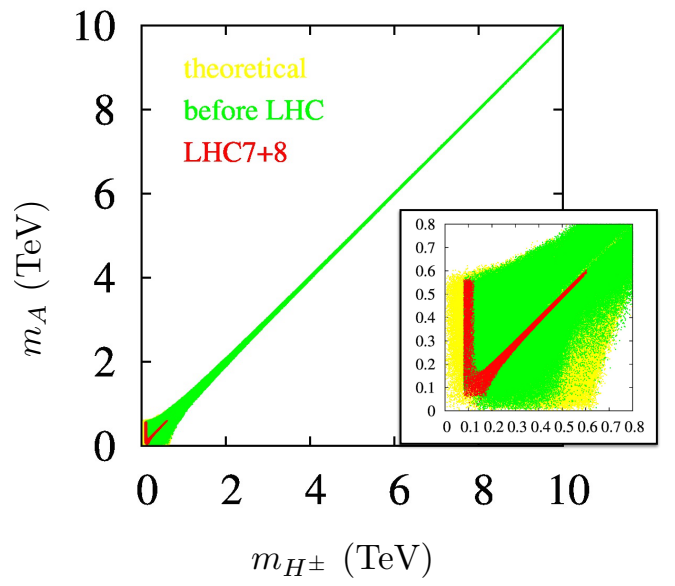

Type-II

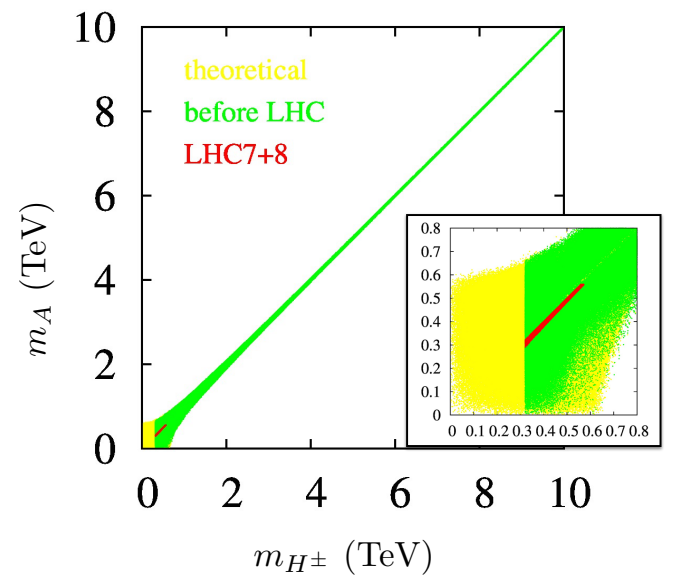

FIG. 1: Constraints in the $m_{A}$ versus $m_{H^{ \pm}}$plane for Type I and Type II 2HDM. Yellow points satisfy theoretical bounds. Green points satisfy up to the pre-LHC constraints while red points satisfy up to LHC constraints. The details of the constraints are summarised in Table I.

normal setup where $h_{125}=h^{0}$, the so-called decoupling limit can be always taken such that all of the new particles are heavy enough to be beyond the LHC reach. A safety zone for avoiding the invalidation of the model by the experimental data is guaranteed. In the hidden light Higgs scenario, however, matching the heavy $H^{0}$ to the $125 \mathrm{GeV}$ state constrains the other heavy Higgs bosons, $A^{0}$ and $H^{ \pm}$.

Figure 1 presents the allowed $m_{A}$ and $m_{H^{ \pm}}$in Type I and Type II. The results for Type X (Type Y) are very similar to Type I (Type II). As summarised in Table I, yellow, green, and red points satisfy theoretical bounds, pre-LHC constraints, and LHC constraints, respectively. Before the LHC data, a band-shaped region of $m_{A} \simeq m_{H^{ \pm}}$is allowed in the $\left(m_{H^{ \pm}}, m_{A}\right)$ space. For Type II, there exist a lower bound on $m_{H^{ \pm}}$, which is constrained by the flavor data, and a lower bound on $m_{A}$ due to the $\Delta \rho$. There are no upper bounds on $m_{A}$ and $m_{H^{ \pm}}$at this stage. Large masses up to $10 \mathrm{TeV}$ are possible, which are dominantly from the $m_{12}^{2}$ terms in Eq. (3). The degeneracy of $m_{A} \approx m_{H^{ \pm}}$is also explained by the same $m_{12}^{2}$ terms of $m_{A}$ and $m_{H^{ \pm}}$, which suppresses the new contribution to the $\Delta \rho$.

When the LHC Higgs data are applied, the most of the green band region is excluded, leaving a very limited parameter space. As shown in the magnified small mass region, the LHC Higgs data put upper bounds on $m_{A}$ and $m_{H^{ \pm}}$. Both should be less than about $600 \mathrm{GeV}$. The strongest bound is from the observed mass of the $125 \mathrm{GeV}$ state with high 


\section{Type II}

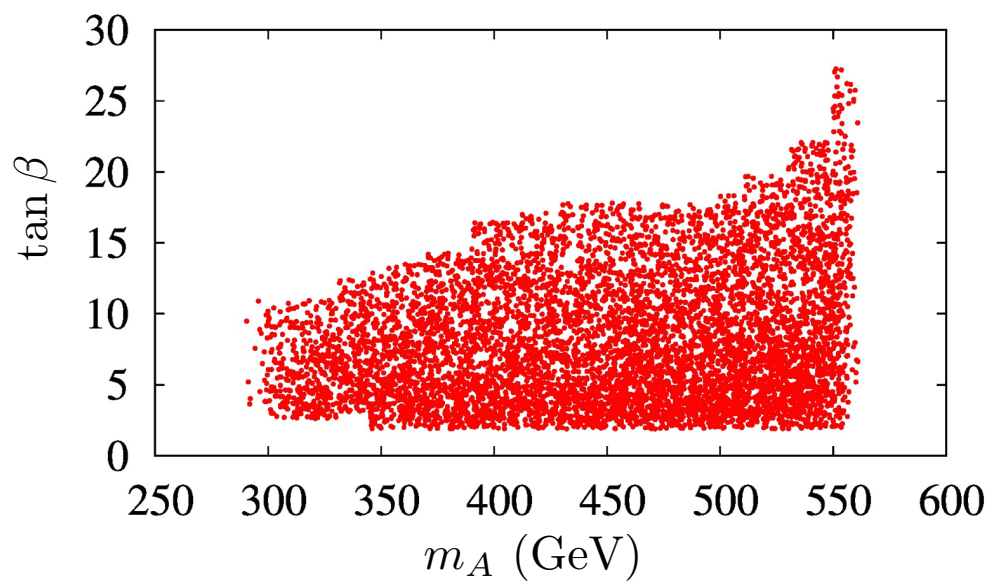

FIG. 2: Allowed parameters by the LHC Higgs data in the plane $\left(m_{A}, t_{\beta}\right)$ for Type II.

precision in Eq. (16). We expect that at the LHC Run 2 both $A^{0}$ and $H^{ \pm}$are to be probed in most of parameter space.

(iii) Strong constraints from the LHC heavy Higgs data in Type II: The 8 TeV LHC heavy Higgs searches in the decay channels of $\gamma \gamma, \tau^{+} \tau^{-}, Z^{0} Z^{0}$, and $W^{+} W^{-}$start to constrain the 2HDM [90]. We find that especially Type II is very sensitive to the results and thus does not allow too large $t_{\beta}$. In Fig. 2, we present the allowed parameters by the LHC Higgs data in the plane $\left(m_{A}, t_{\beta}\right)$ for Type II. The heavy Higgs search results remove two regions, small $t_{\beta}$ region with $m_{A} \lesssim 2 m_{t}$ and large $t_{\beta}$ region.

The small $t_{\beta}$ region is excluded by the $\gamma \gamma$ channel through the gluon fusion production, similarly to the $h^{0}=h_{125}$ case [60]. Both $g-g-A^{0}$ and $\gamma-\gamma-A^{0}$ vertices are loop induced, mainly through the top quark loop. Since all of four types have top quark Yukawa couplings with $A^{0}$ be inversely proportional to $t_{\beta}$, small $t_{\beta}$ yields sizable gluon fusion production as well as sizable branching ratio into $\gamma \gamma$. If $m_{A}=330 \mathrm{GeV}$, for example, $t_{\beta}$ should be above 2.5. This is stronger than the constraints from $b \rightarrow s \gamma$ and $\Delta M_{B_{d}}$ on small $t_{\beta}$ like $t_{\beta} \gtrsim 1$ although the FCNC bound depends on the charged Higgs boson mass. Note that the exclusion of this small $t_{\beta}$ region is common for all four types. As $m_{A}$ goes beyond the $t \bar{t}$ threshold, the main decay mode of $A^{0}$ is into $t \bar{t}$, with the branching ratio practically one unless $t_{\beta}$ is too large.

In Type II, large $t_{\beta}$ region is excluded mainly by $b \bar{b} \rightarrow A^{0} \rightarrow \tau^{+} \tau^{-}[90$. Here, both $b$ quark and $\tau$ Yukawa couplings with $A^{0}$ are proportional to $t_{\beta}$, yielding the signal rate proportional to $t_{\beta}^{4}$. A large portion of the parameter space is excluded. For example, the 
Type I

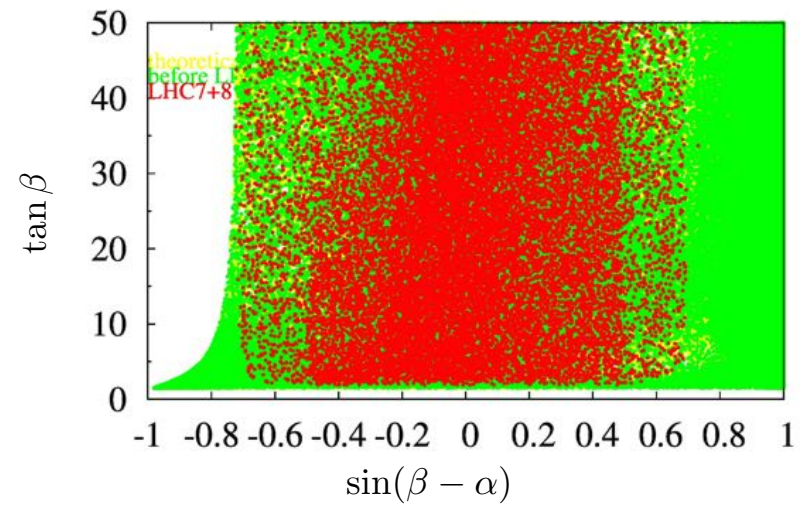

Type X

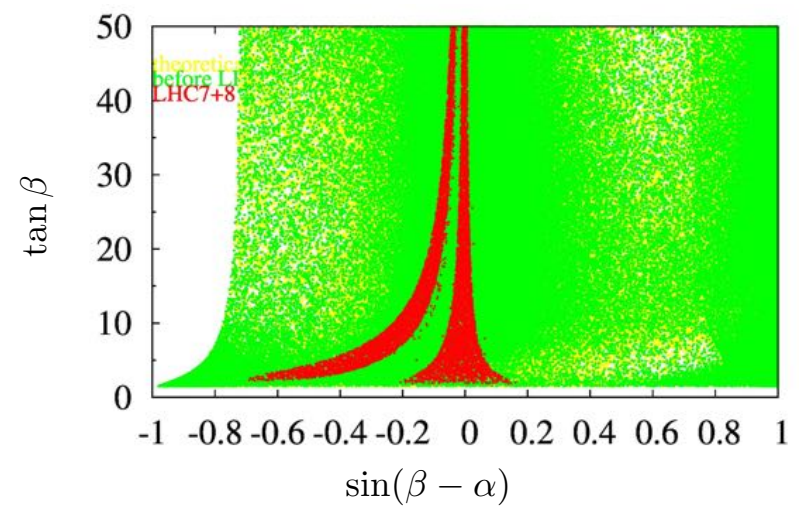

Type II

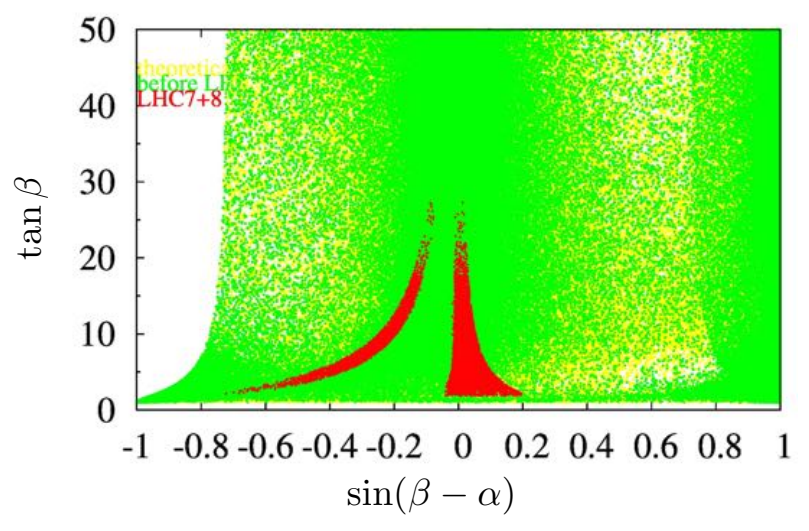

Type Y

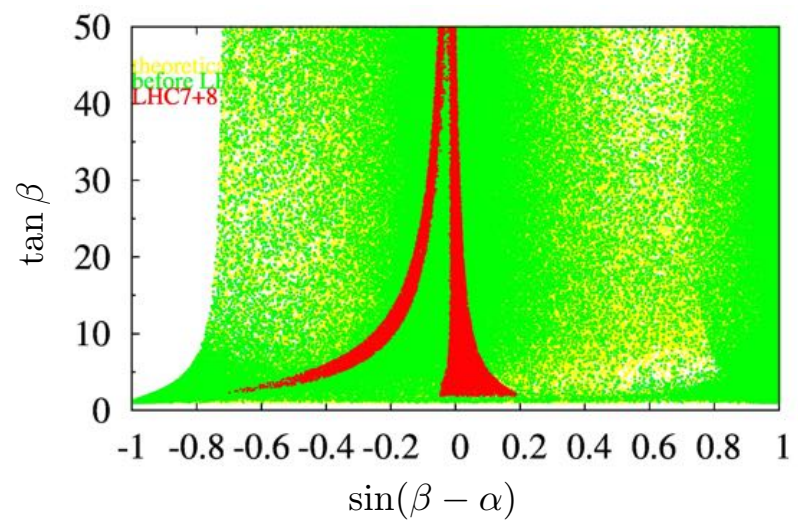

FIG. 3: Constraints in the $(\sin (\beta-\alpha), \tan \beta)$ plane for Type I, II, X, and Y. Color scheme is the same as in Fig. 1 .

$m_{A}=300 \mathrm{GeV}$ case excludes $t_{\beta} \gtrsim 10$. In Type $\mathrm{X}$ and Type $\mathrm{Y}$, the multiplication of $\tau$ and $b$ quark Yukawa couplings with $A^{0}$ does not have $t_{\beta}$ dependence. The LHC $8 b \bar{b} \rightarrow A^{0} \rightarrow \tau^{+} \tau^{-}$ constraint for Types $\mathrm{X}$ and $\mathrm{Y}$ is not strong yet. In Type I, all of the Yukawa couplings are suppressed by $t_{\beta}$, which is weakly constrained.

(iv) Preferring the alignment limit: The deviation from the alignment limit is well parameterised by $s_{\beta-\alpha}$. In Fig. 3 , we show the allowed parameter space in the $\left(s_{\beta-\alpha}, t_{\beta}\right)$ plane for Type I, II, X, and Y. Type I allows sizable deviation: $\left|s_{\beta-\alpha}\right| \lesssim 0.5$ in most cases, but even $\left|s_{\beta-\alpha}\right| \simeq 0.7$ is allowed scarcely. This is expected from the normalised Yukawa couplings in Eq. (10) which have the $t_{\beta}$-suppressed deviation from the alignment. The constraint from the LHC Higgs data on $s_{\beta-\alpha}$ is relatively weak. For other types, the $t_{\beta}$-enhanced Yukawa couplings constrain the model, resulting in the preference to the alignment limit, stronger for large $t_{\beta}$. 


\section{Type I}

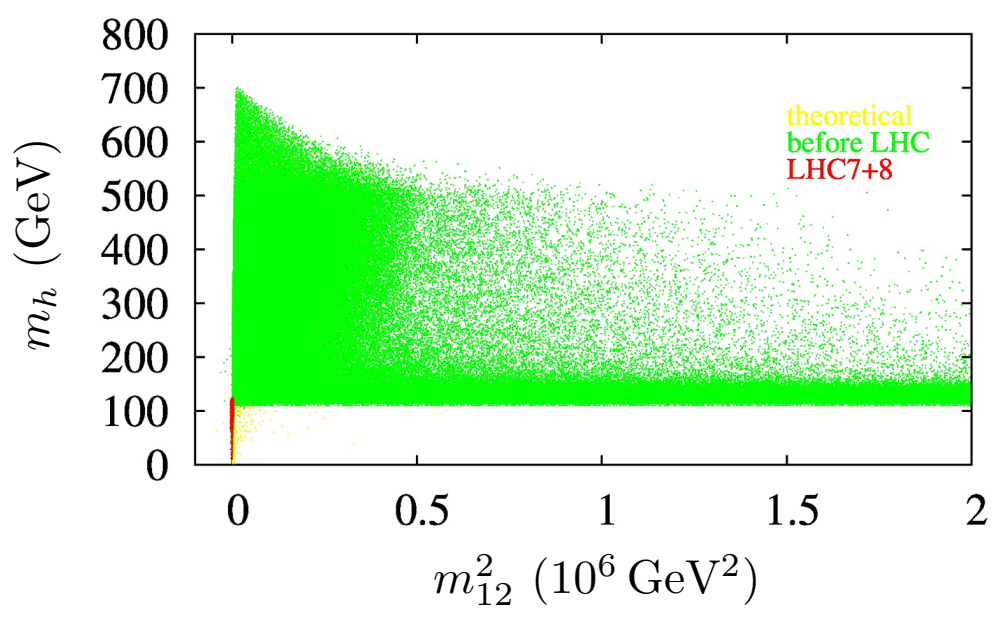

FIG. 4: Constraints in the $\left(m_{12}^{2}, m_{h}\right)$ plane for Type I. Color scheme is the same as in Fig. 1.

For Type II, X, and Y, there are two separate regions of allowed parameters. One region is along the alignment line $\left(s_{\beta-\alpha}=0\right)$. For small $t_{\beta}$ which weakens the $t_{\beta}$-enhanced deviations in Eq. 10, $s_{\beta-\alpha}$ can be as large as 0.2. The other type of the allowed regions is a bit apart from the $s_{\beta-\alpha}=0$ line but toward negative $s_{\beta-\alpha}$. For small $t_{\beta}, s_{\beta-\alpha}$ can be as large as -0.6 . As shown in Fig. 2, Type II does not have the allowed region for $t_{\beta}>20$ for the most part.

(v) Low scale for $m_{12}^{2}$ : The $m_{12}^{2}$ term in the Higgs potential breaks the $Z_{2}$ symmetry softly. The explicit soft $Z_{2}$ breaking is to be understood as the result of spontaneous symmetry breaking in a more fundamental theory. Without a knowledge of the origin and dynamics of the $Z_{2}$ breaking, there is no guideline about the $m_{12}^{2}$ scale. In the hidden light Higgs scenario, however, the condition of $m_{H}=125 \mathrm{GeV}$ constrains the $m_{12}^{2}$ scale very strongly. It is clearly shown in Fig. 4 by the allowed parameters in the $\left(m_{12}^{2}, m_{h}\right)$ plane for Type I. Before the LHC data, $m_{12}^{2}$ is not limited, while the LEP bounds exclude most of the parameter space for $m_{h}<114 \mathrm{GeV}$. The current LHC Higgs data (red points) do prefer the low scale of $m_{12}^{2}$, which implies that the $Z_{2}$ parity is a good approximate symmetry in the scenario.

In Fig. 5, we show the constraints in the $\left(m_{12}^{2}, m_{h}\right)$ plane for Type I, II, X, and Y, focused on the LHC allowed regions. All of the four types show similar shapes of the allowed regions: one exception is Type II where the allowed space is much smaller. As discussed before, this is because of the strong constraint from the heavy Higgs boson search in the $b$ quark associated production followed by the decay into $\tau^{+} \tau^{-}$. Positive $m_{12}^{2}$ is preferred, although 
Type-I

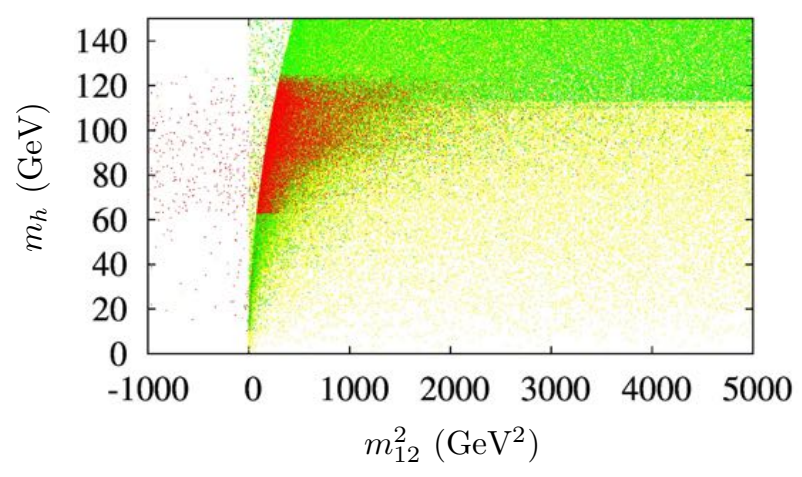

Type-X

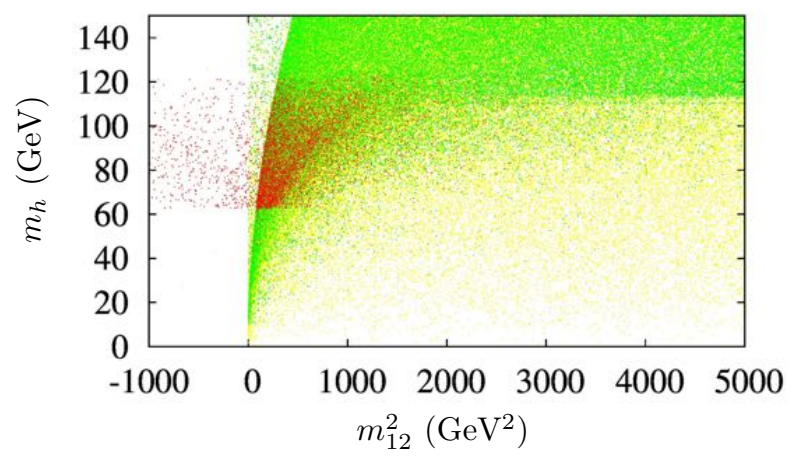

Type-II

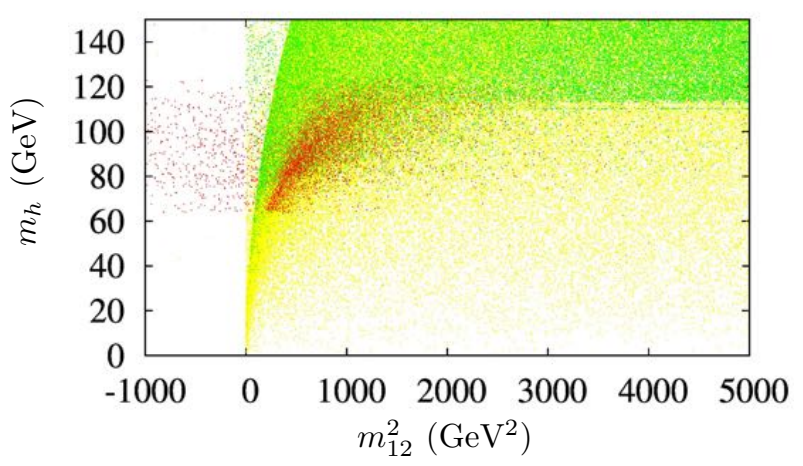

Type-Y

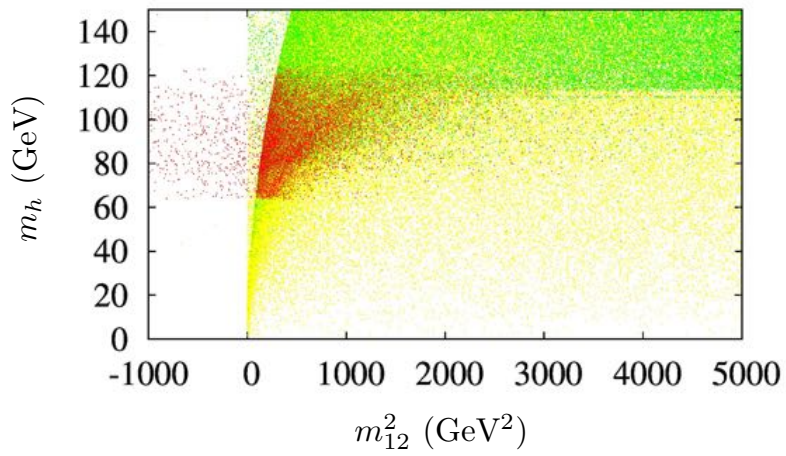

FIG. 5: Constraints in the $\left(m_{12}^{2}, m_{h}\right)$ plane for Type I and Type II. Color scheme is the same as in Fig. 1 .

small negative value is also allowed. The magnitude of $\left|m_{12}\right|$ is below about $45 \mathrm{GeV}$ mostly.

Note that very light $h^{0}$ is possible in Type I. However, too light scalar bosons may cause dangerous decay models such as $\eta \rightarrow \pi h^{0}, \Upsilon \rightarrow A^{0} \gamma$, and $J / \Psi \rightarrow A^{0} \gamma$. Although very light scalar bosons with mass below $10 \mathrm{GeV}$ are not excluded in some parameter space [91 93], we require for both $h^{0}$ and $A^{0}$ to be heavier than $10 \mathrm{GeV}$ for simplicity. Another important constraint is from the $H^{0} \rightarrow h^{0} h^{0}$ decay, which affects the LHC Higgs signal strength measurement. In the alignment limit, the $H^{0}-h^{0}-h^{0}$ vertex, normalized by the SM vertex $g_{h h h}^{\mathrm{SM}}=3 m_{h_{\mathrm{SM}}}^{2} / v$, is

$$
\hat{g}_{H h h}=\frac{1}{3}\left[1+2 \frac{m_{h}^{2}}{m_{H}^{2}}-2\left(t_{\beta}+\frac{1}{t_{\beta}}\right) \frac{m_{12}^{2}}{m_{H}^{2}}\right]+\mathcal{O}\left(s_{\beta-\alpha}\right) .
$$

In the alignment limit, the decay $H^{0} \rightarrow h^{0} h^{0}$ is sizable in general. In Types II, X, and Y where the alignment limit is strongly preferred (see Fig. 3), $\hat{g}_{H h h}$ is too large to accommodate the LHC Higgs data unless $m_{12}^{2}$ and $t_{\beta}$ are tuned to suppress $\hat{g}_{H h h}$. It turns out that the 
Type I

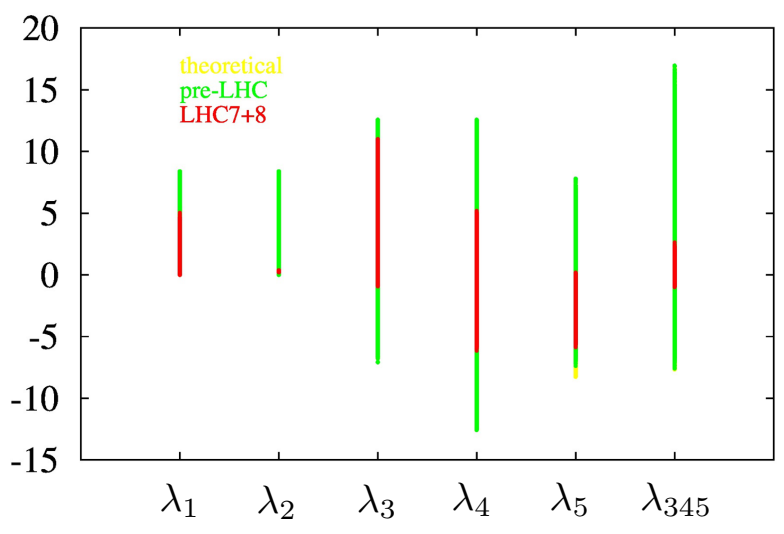

Type X

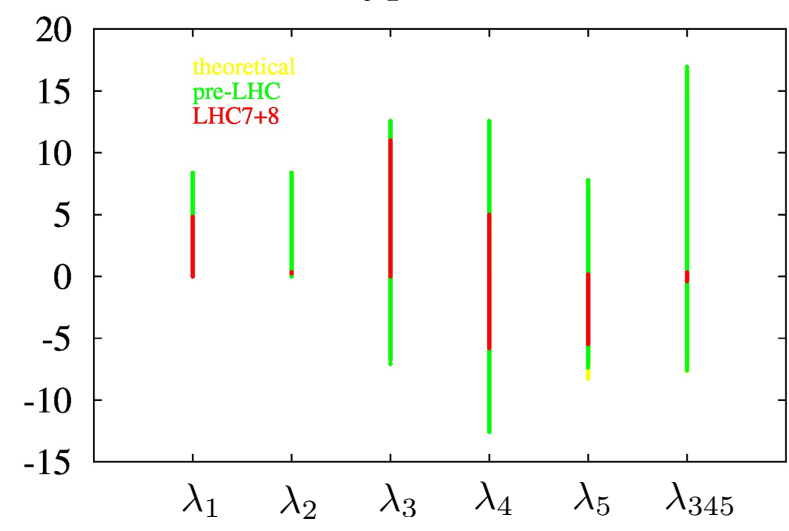

Type II

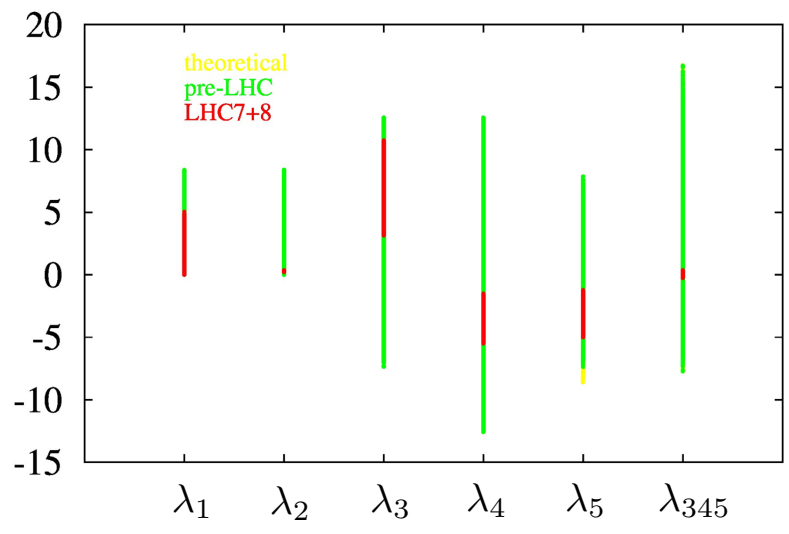

Type $\mathbf{Y}$

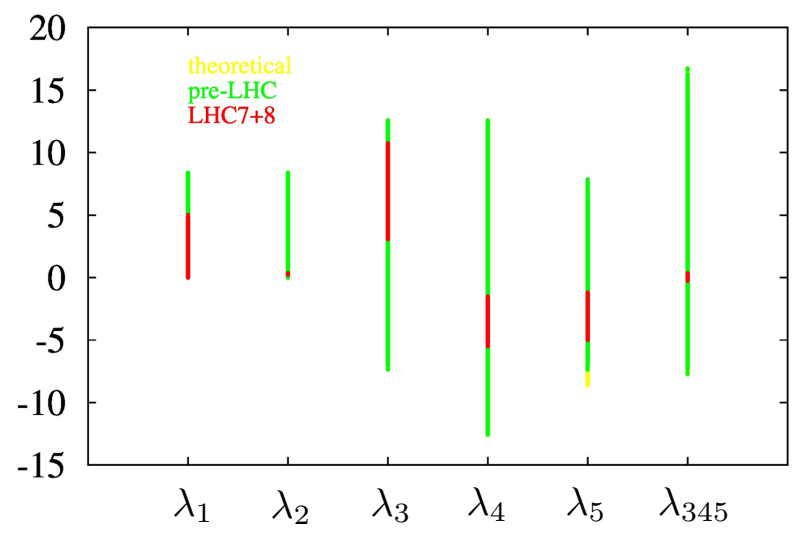

FIG. 6: Constraints on $\lambda_{1,2,3,4,5}$ and $\lambda_{345}=\lambda_{3}+\lambda_{4}+\lambda_{5}$ for Type I, II, X, and Y. Color scheme is the same as in Fig. 1.

LHC Higgs data prefer $m_{h}>m_{H} / 2$ in Type II, X, and Y in a general analysis based on the random generation of parameter points. On the other hand, a very light Higgs boson with $m_{h}<m_{H} / 2$ is allowed, though less probable, when we carry out the analysis by choosing the special parameter choices leading to small enough $\hat{g}_{H h h}$ as shown in Ref. [31].

(vi) Strong bound on $\lambda_{2}$ but weak bounds on $\lambda_{1,3,4,5}$ : Figure 6 shows how much the current LHC Higgs data constrain the Higgs potential parameters $\lambda_{i}$ 's. For all of four types, $\lambda_{2}$ is almost determined to be around 0.26 . This is mainly by the mass measurement of $m_{H}=$ $125 \mathrm{GeV}$. Since $m_{12}^{2}$ is small (see Fig. 5) and $t_{\beta}>1$ from the FCNC constraints, we have $m_{H} \sim \sqrt{\lambda_{2}} v$ in the large $t_{\beta}$ limit: see Eqs. (5) and (6). The condition of $m_{H}=125 \mathrm{GeV}$ almost fixes $\lambda_{2}$. Secondly, $\lambda_{345}\left(\equiv \lambda_{3}+\lambda_{4}+\lambda_{5}\right)$ is also limited by the observed Higgs boson mass in Types II, X, and Y. The other $\lambda_{i}$ 's are not seriously constrained. Compared to the 
triple couplings(Type-I)

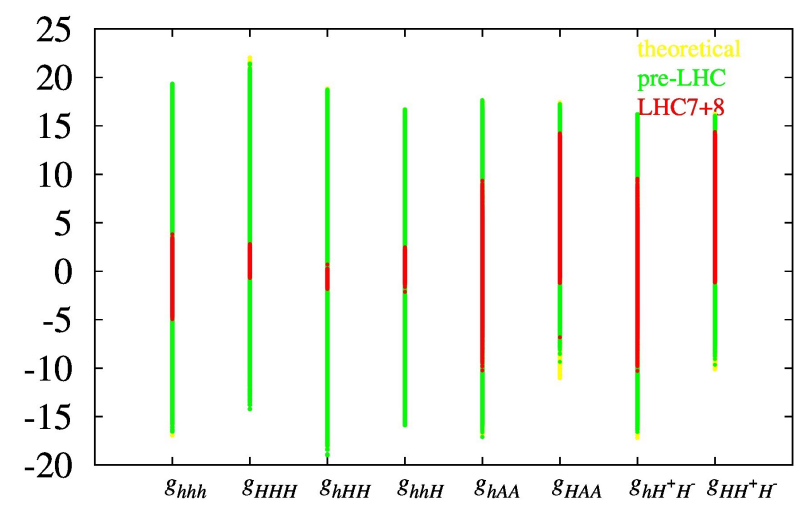

triple couplings(Type-X)

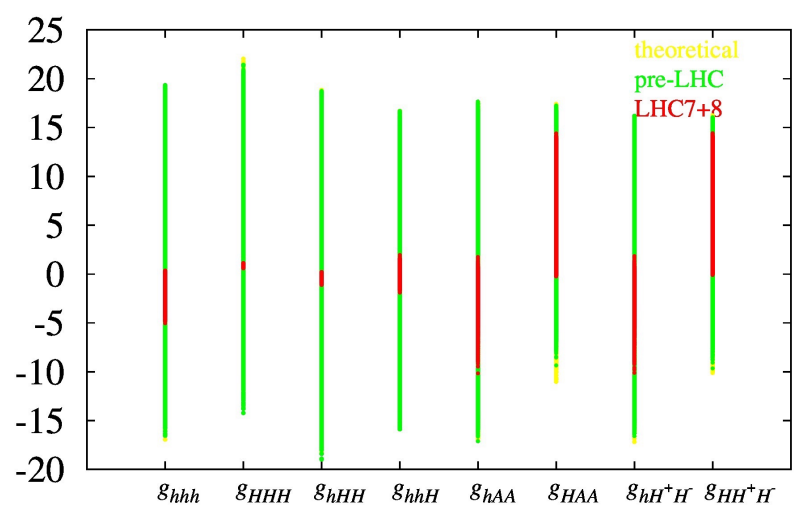

triple couplings(Type-II)
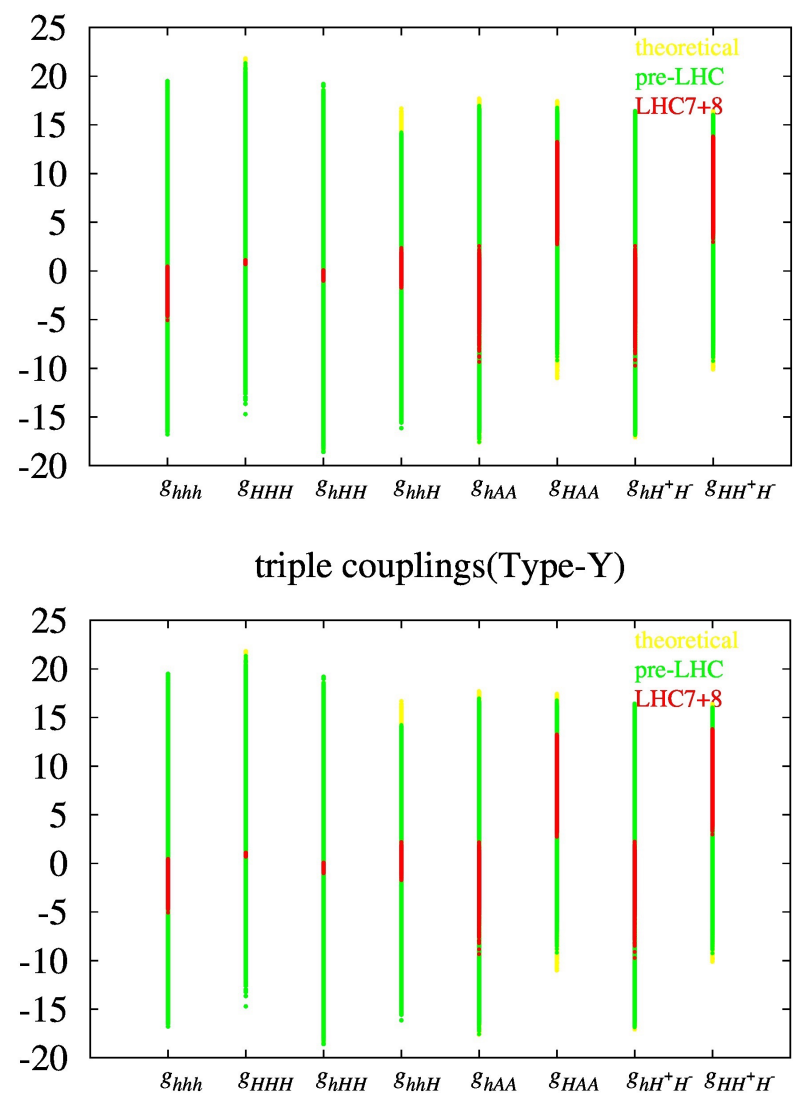

FIG. 7: Constraints on $\hat{g}_{\phi_{i} \phi_{j} \phi_{k}}$ for Type I, II, X, and Y. Color scheme is the same as in Fig. 1.

pre-LHC (green) situation, the LHC Higgs data reduce the value of $\lambda_{1,3,4,5}$ by about half. Considering the role of the current LHC Higgs signal strength measurement in determining $\lambda_{2}$, we anticipate that the values of $\lambda_{i}$ would be substantially reduced if additional Higgs bosons are observed.

\section{FUTURE PROSPECT OF $e^{+} e^{-} \rightarrow Z^{0} H^{0} H^{0}$}

Focusing on the determination of the Higgs potential in the hidden light Higgs scenario, we study the current status of the Higgs triple and quartic couplings. First we study the allowed range of various Higgs triple couplings. In the normal setup with $h_{125}=h^{0}$, Ref. [94] showed that the LHC Higgs data constrain the normalised Higgs triple couplings $\hat{g}_{h h h}$ lies between 0.56 and 1 at 95\% C.L. level in Type II. The Higgs boson pair production at a 14 TeV LHC was calculated for some benchmarks, not for the whole allowed parameter space, 
Type-I; quartic couplings

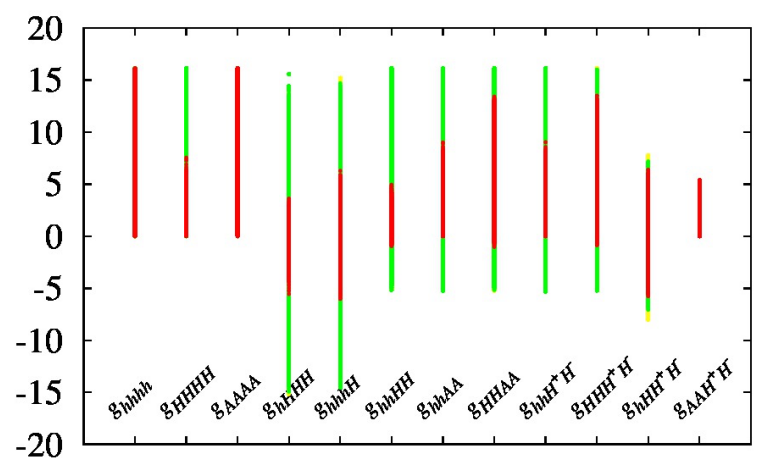

Type-X; quartic couplings

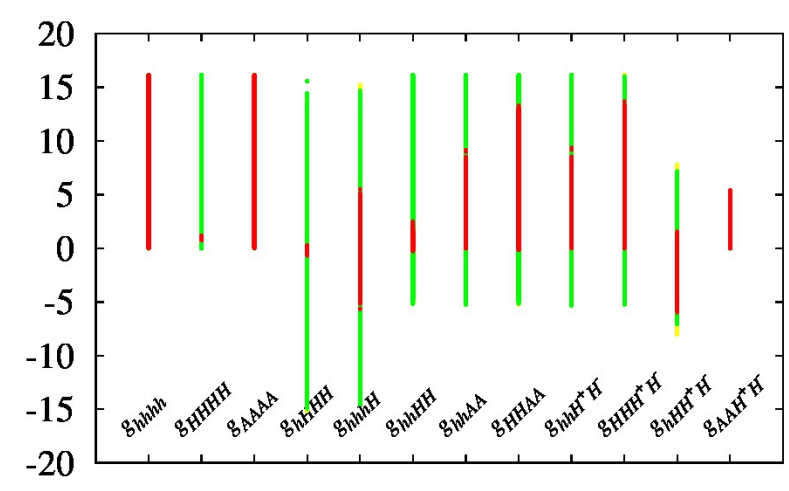

Type-II; quartic couplings

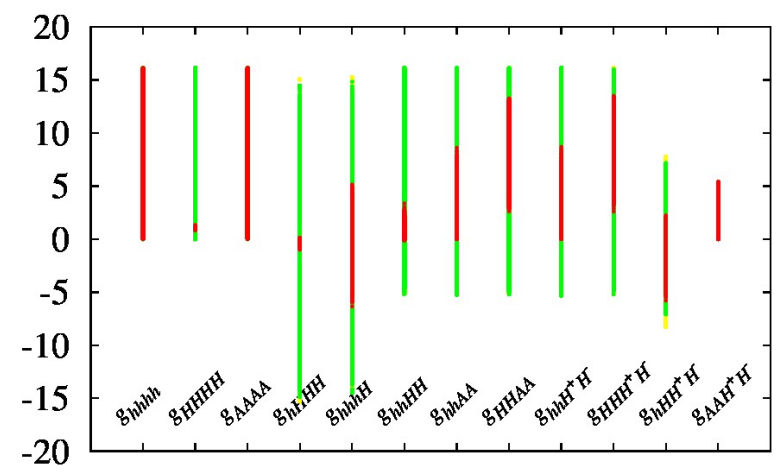

Type-Y; quartic couplings

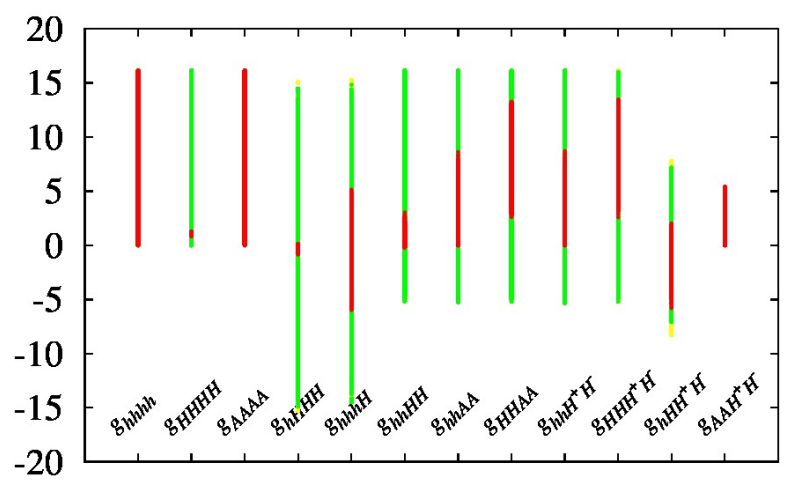

FIG. 8: Constraints on $\hat{g}_{\phi_{i} \phi_{j} \phi_{k} \phi_{\ell}}$ for Type I, II, X, and Y. Color scheme is the same as in Fig. 1 .

since the physical properties of the heavy Higgs bosons are weakly constrained in the normal setup. In the hidden light Higgs scenario, the heavy Higgs boson properties are shown to be significantly limited, which may results in higher predictability in the process involving the Higgs triple couplings.

In Fig. 7, we present the allowed values of the normalised Higgs triple couplings, $\hat{g}_{\phi_{i} \phi_{j} \phi_{k}}$ for $\phi_{i}=h^{0}, H^{0}, A^{0}, H^{ \pm}$. For all of four types, the triple coupling involving two $125 \mathrm{GeV}$ states, $\hat{g}_{h H H}$ and $\hat{g}_{H H H}$, are quite strongly constrained. Compared to the pre-LHC constraint, the LHC data restrict $\hat{g}_{h H H}$ and $\hat{g}_{H H H}$ within a few percent. In particular, $\hat{g}_{H H H}$ in Types II, X, and $\mathrm{Y}$ are very limited between 0.69 and 1.1. In Type I that allows much larger parameter space, the reduction is into $10 \%$ level.

The quartic Higgs couplings are in general less constrained even in the hidden light Higgs scenario. In Fig. 8, we show the normalized quartic couplings by the SM value, with the three step constraints imposed. It is remarkable that $\hat{g}_{H H H H}$ and $\hat{g}_{h H H H}$ in Types II, X, 

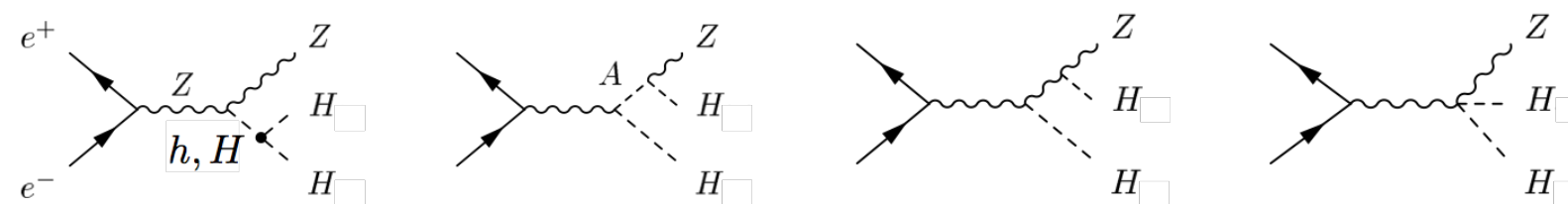

FIG. 9: Feynman diagrams for $e^{+} e^{-} \rightarrow Z^{0} H^{0} H^{0}$.

and Y are quite significantly constrained as to be similar to the SM value. On the contrary, Type I does not have limited value of $\hat{g}_{H н H н}$ and $\hat{g}_{h H H H}$ because of the large allowed parameter space. Other quartic Higgs couplings can be very large compared with the SM value. Contrary to the SM case where the quartic coupling will remain unaccessible due to the tiny cross section of $e^{+} e^{-} \rightarrow Z^{0} h_{\mathrm{SM}} h_{\mathrm{SM}} h_{\mathrm{SM}}$ [11, 12, some large quartic couplings can yield large enough cross section. In all of the four types, $\hat{g}_{h h h h}$ and $\hat{g}_{A A A A}$ can have the enhancement factor more than ten, which can be probed, e,g, through $e^{+} e^{-} \rightarrow Z^{0} h^{0} h^{0} h^{0}$ and $\gamma \gamma \rightarrow A^{0} A^{0} A^{0}$, respectively.

In order to probe $\hat{g}_{h H H}$ and $\hat{g}_{H H H}$, we study the double Higgs-strahlung at an $e^{+} e^{-}$ collider, $e^{+} e^{-} \rightarrow Z^{0} H^{0} H^{0}$ [95] 97]. The Feynman diagrams are presented in Fig. 9. Since the physical properties of $H^{0}$ are well known, the Higgs boson can be used as a tagging particle for a new physics model. $\hat{g}_{h H H}$ and $\hat{g}_{H H H}$ contribute in the first diagram. The second Feynman diagram shows that the $Z^{0}-H^{0}-A^{0}$ vertex also contributes. As classified in Eq. (9), the $Z^{0}-H^{0}-A^{0}$ vertex is proportional to $s_{\beta-\alpha}$, which is suppressed in the alignment limit. If $s_{\beta-\alpha} \neq 0$ and the kinematical space includes the pole of the $A^{0}$ propagator, the total cross section can be highly enhanced.

Figure 10 shows the expected total cross section of $e^{+} e^{-} \rightarrow Z^{0} H^{0} H^{0}$ versus $m_{A}$ at $\sqrt{s}=500 \mathrm{GeV}$, normalized by the $\mathrm{SM}$ cross section. We accept only the parameter points which satisfy all of the current constraints. The analytic expression for $\sigma\left(e^{+} e^{-} \rightarrow Z^{0} H^{0} H^{0}\right)$ is referred to Ref. [97]. In most parameter space, the cross section in the 2HDM is very similar to that in the SM. In some parameter space, however, all of the four types allow highly enhanced cross section. The rate of increase can be as large as factor of $10^{4}$. As clearly shown in Types I and X, the enhancement occurs when $m_{A} \geq m_{Z}+m_{H}$. 

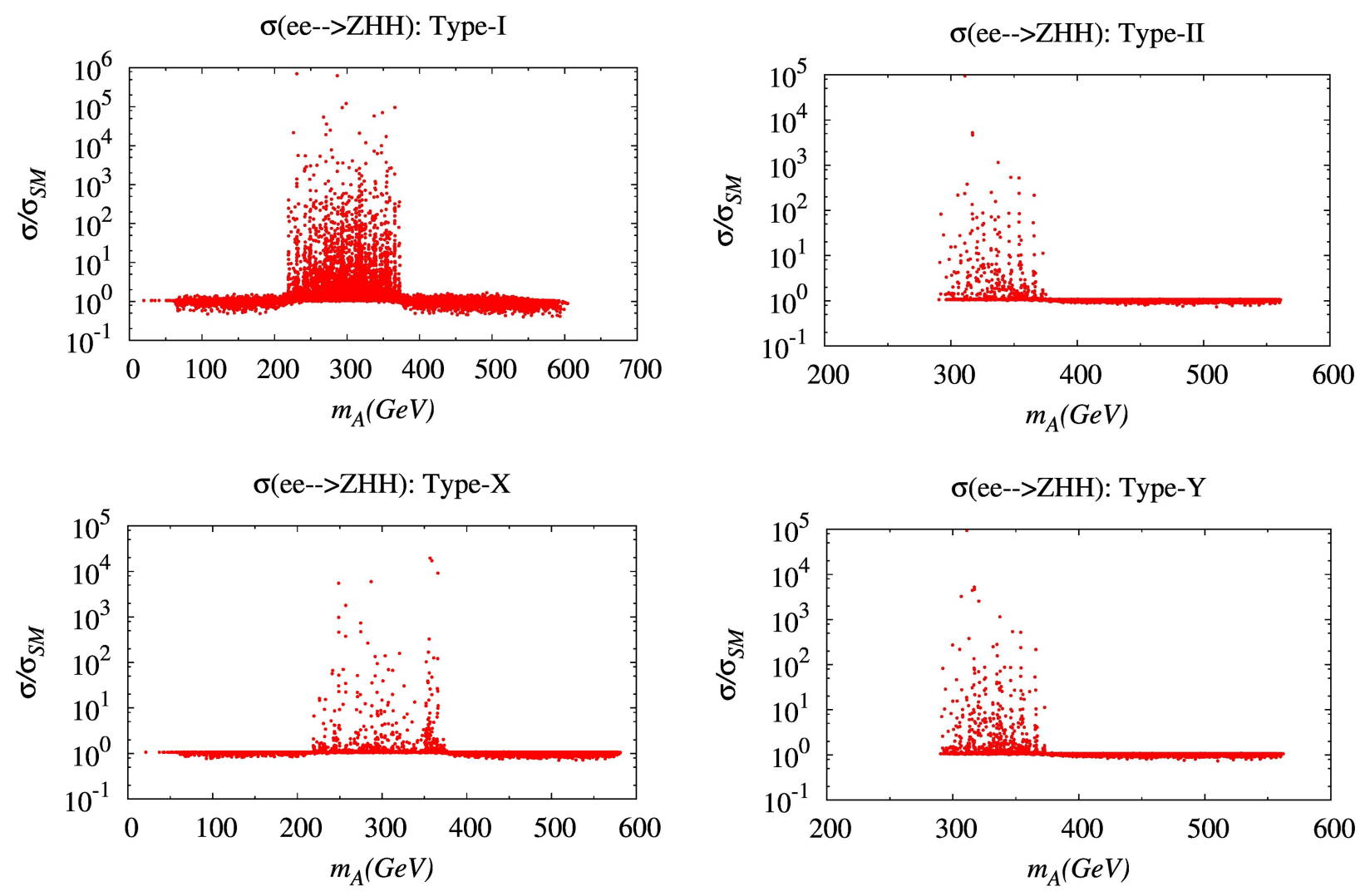

FIG. 10: The cross section of $e^{+} e^{-} \rightarrow Z^{0} H^{0} H^{0}$ versus $m_{A}$ at $\sqrt{s}=500 \mathrm{GeV}$, normalized by the SM cross section, for the parameter points satisfying the LHC Higgs data.

\section{CONCLUSIONS}

We have investigated the meaning of the LHC Run 1 in the context of the hidden light Higgs scenario in the $2 \mathrm{HDM}$ with $C P$ invariance and the softly broken $Z_{2}$ symmetry. We found that the LHC Run 1 data combined with other current constraints do not exclude the possibility that the observed scalar particle is the heavier $C P$-even Higgs boson $H^{0}$. The lighter $C P$-even Higgs boson $h^{0}$ is buried in the region of $\lesssim 120 \mathrm{GeV}$. A remarkable consequence is that in order to make $m_{H}=125 \mathrm{GeV}$ the $Z_{2}$ symmetry breaking parameter $m_{12}^{2}$ cannot be large, which renders $m_{A, H^{ \pm}}$rather light at the sub-TeV scale. We found the upper bounds on $m_{A, H^{ \pm}}$to be around $600 \mathrm{GeV}$. Since the mass scale of other Higgs bosons are not far from the LHC reach, the hidden light Higgs scenario can be tested in the near future.

We also found that the LHC Run 1 data begin to constrain the Higgs potential of the 2HDM. In particular, the values of $\lambda_{2}$ and $\hat{g}_{H H H}$ are almost determined. The cross section 
of $e^{+} e^{-} \rightarrow Z^{0} H^{0} H^{0}$ is expected to be close to that of the SM, while in a limited region of $m_{A}$ around $300 \mathrm{GeV}$ it could be highly enhanced. The Higgs quartic couplings are less constrained. Hopefully future lepton colliders could check our predictions.

\section{Acknowledgments}

SKK was supported by the National Research Foundation of Korea, NRF2014R1A1A2057665. JPL was supported by the second stage of the Brain Korea 21 Plus Project in 2015. JS was supported by the National Research Foundation of Korea, NRF2013R1A1A2061331. We thank Convergence Computing team of National Institute for Mathematical Sciences for valuable comments in extracting data from experimental papers.

[1] G. Aad et al. [ATLAS Collaboration], Phys. Lett. B 716, 1 (2012); S. Chatrchyan et al. [CMS Collaboration], Phys. Lett. B 716, 30 (2012).

[2] CMS Collaboration [CMS Collaboration], CMS-PAS-HIG-14-009; G. Aad et al. [ATLAS Collaboration], Phys. Rev. D 90, no. 5, 052004 (2014) arXiv:1406.3827 [hep-ex]].

[3] G. Aad et al. [ATLAS Collaboration], Phys. Rev. D 90, no. 11, 112015 (2014) arXiv:1408.7084 [hep-ex]].

[4] G. Aad et al. [ATLAS Collaboration], Phys. Lett. B 740, 222 (2015) arXiv:1409.3122 [hep$\mathrm{ex}]$.

[5] V. Khachatryan et al. [CMS Collaboration], Eur. Phys. J. C 74, no. 10, 3076 (2014) arXiv:1407.0558 [hep-ex]].

[6] H. S. Cheon and S. K. Kang, JHEP 1309, 085 (2013); S. Chang, S. K. Kang, J. P. Lee, K. Y. Lee, S. C. Park and J. Song, JHEP 1305, 075 (2013); N. Chakrabarty, U. K. Dey and B. Mukhopadhyaya, JHEP 1412, 166 (2014) arXiv:1407.2145 [hep-ph]].

[7] S. Chang, S. K. Kang, J. P. Lee, K. Y. Lee, S. C. Park and J. Song, JHEP 1409, 101 (2014).

[8] J. F. Gunion and H. E. Haber, Phys. Rev. D 67, 075019 (2003) hep-ph/0207010].

[9] A. Noble and M. Perelstein, Phys. Rev. D 78, 063518 (2008) arXiv:0711.3018 [hep-ph]].

[10] A. Katz and M. Perelstein, JHEP 1407, 108 (2014) arXiv:1401.1827 [hep-ph]].

[11] M. Battaglia, E. Boos and W. M. Yao, eConf C 010630, E3016 (2001) hep-ph/0111276. 
[12] M. Beyer, W. Kilian, P. Krstonosic, K. Monig, J. Reuter, E. Schmidt and H. Schroder, Eur. Phys. J. C 48, 353 (2006) hep-ph/0604048.

[13] J. F. Gunion and H. E. Haber, Phys. Rev. D 67, 075019 (2003); G. C. Branco, P. M. Ferreira, L. Lavoura, M. N. Rebelo, M. Sher and J. P. Silva, Phys. Rept. 516, 1 (2012).

[14] S. L. Glashow and S. Weinberg, Phys. Rev. D 15, 1958 (1977); E. A. Paschos, Phys. Rev. D 15, 1966 (1977).

[15] M. Aoki, S. Kanemura, K. Tsumura and K. Yagyu, Phys. Rev. D 80, 015017 (2009).

[16] A. G. Akeroyd, Phys. Lett. B 377, 95 (1996) [hep-ph/9603445].

[17] C. Y. Chen and S. Dawson, Phys. Rev. D 87, 055016 (2013) arXiv:1301.0309 [hep-ph]].

[18] C. W. Chiang and K. Yagyu, JHEP 1307, 160 (2013) arXiv:1303.0168 [hep-ph]].

[19] B. Grinstein and P. Uttayarat, JHEP 1306, 094 (2013) arXiv:1304.0028 [hep-ph]].

[20] O. Eberhardt, U. Nierste and M. Wiebusch, JHEP 1307, 118 (2013) arXiv:1305.1649 [hep$\mathrm{ph}]$.

[21] G. Belanger, B. Dumont, U. Ellwanger, J. F. Gunion and S. Kraml, Phys. Rev. D 88, 075008 (2013) arXiv:1306.2941 [hep-ph]].

[22] K. Cheung, J. S. Lee and P. Y. Tseng, JHEP 1401, 085 (2014) [arXiv:1310.3937 [hep-ph]].

[23] A. Celis, V. Ilisie and A. Pich, JHEP 1312, 095 (2013) arXiv:1310.7941 [hep-ph]].

[24] L. Wang and X. F. Han, JHEP 1404, 128 (2014) arXiv:1312.4759 [hep-ph]].

[25] S. Kanemura, K. Tsumura, K. Yagyu and H. Yokoya, Phys. Rev. D 90, 075001 (2014) arXiv:1406.3294 [hep-ph]].

[26] P. M. Ferreira, R. Guedes, J. F. Gunion, H. E. Haber, M. O. P. Sampaio and R. Santos, arXiv:1407.4396 [hep-ph].

[27] A. Broggio, E. J. Chun, M. Passera, K. M. Patel and S. K. Vempati, JHEP 1411, 058 (2014) arXiv:1409.3199 [hep-ph]].

[28] D. Chowdhury and O. Eberhardt, arXiv:1503.08216 [hep-ph].

[29] L. Wang and X. F. Han, JHEP 1411, 085 (2014) arXiv:1404.7437 [hep-ph]].

[30] S. Kanemura, H. Yokoya and Y. J. Zheng, Nucl. Phys. B 886, 524 (2014) arXiv:1404.5835 [hep-ph]].

[31] J. Bernon, J. F. Gunion, Y. Jiang and S. Kraml, Phys. Rev. D 91, 075019 (2015) arXiv:1412.3385 [hep-ph]].

[32] B. Coleppa, F. Kling and S. Su, JHEP 1401, 161 (2014) arXiv:1305.0002 [hep-ph]]. 
[33] E. Cervero and J. M. Gerard, Phys. Lett. B 712, 255 (2012) arXiv:1202.1973 [hep-ph]].

[34] S. de Visscher, J. M. Gerard, M. Herquet, V. Lemaitre and F. Maltoni, JHEP 0908, 042 (2009) arXiv:0904.0705 [hep-ph]].

[35] P. M. Ferreira, R. Guedes, M. O. P. Sampaio and R. Santos, JHEP 1412, 067 (2014) arXiv:1409.6723 [hep-ph]].

[36] B. Dumont, J. F. Gunion, Y. Jiang and S. Kraml, Phys. Rev. D 90, 035021 (2014) arXiv:1405.3584 [hep-ph]].

[37] B. Bhattacherjee, A. Chakraborty and A. Choudhury, arXiv:1504.04308 [hep-ph].

[38] I. P. Ivanov, Phys. Rev. D 75, 035001 (2007) [Erratum-ibid. D 76, 039902 (2007)] hepph/0609018.

[39] A. Arhrib, hep-ph/0012353.

[40] G. C. Branco, P. M. Ferreira, L. Lavoura, M. N. Rebelo, M. Sher and J. P. Silva, Phys. Rept. 516, 1 (2012) arXiv:1106.0034 [hep-ph]].

[41] A. Sopczak, hep-ph/0502002.

[42] R. Barate et al. [LEP Working Group for Higgs boson searches and ALEPH and DELPHI and L3 and OPAL Collaborations], Phys. Lett. B 565, 61 (2003); S. Schael et al. [ALEPH and DELPHI and L3 and OPAL and LEP Working Group for Higgs Boson Searches Collaborations], Eur. Phys. J. C 47, 547 (2006).

[43] K. A. Olive et al. [Particle Data Group Collaboration], Chin. Phys. C 38, 090001 (2014).

[44] John F. Gunion, Howard E. Haber, Gordon Kane, Sally Dawson, The Higgs Hunter's Guide, Addison-Wesley Publishing Company, 1990.

[45] P. H. Chankowski, M. Krawczyk and J. Zochowski, Eur. Phys. J. C 11, 661 (1999).

[46] W. S. Hou and R. S. Willey, Phys. Lett. B 202, 591 (1988); B. Grinstein and M. B. Wise, Phys. Lett. B 201, 274 (1988); B. Grinstein, R. P. Springer and M. B. Wise, Nucl. Phys. B 339, 269 (1990); V. D. Barger, J. L. Hewett and R. J. N. Phillips, Phys. Rev. D 41, 3421 (1990); S. Bertolini, F. Borzumati, A. Masiero and G. Ridolfi, Nucl. Phys. B 353, 591 (1991).

[47] F. Mahmoudi and O. Stal, Phys. Rev. D 81, 035016 (2010).

[48] A. Abulencia et al. [CDF Collaboration], Phys. Rev. Lett. 96, 042003 (2006) hep-ex/0510065; V. M. Abazov et al. [D0 Collaboration], Phys. Rev. Lett. 88, 151803 (2002) [hep-ex/0102039].

[49] G. Aad et al. [ATLAS Collaboration], JHEP 1503, 088 (2015) arXiv:1412.6663 [hep-ex]]; The ATLAS collaboration, ATLAS-CONF-2013-090, ATLAS-COM-CONF-2013-107. 
[50] CMS Collaboration [CMS Collaboration], CMS-PAS-HIG-14-020; S. Chatrchyan et al. [CMS Collaboration], JHEP 1207, 143 (2012) arXiv:1205.5736 [hep-ex]].

[51] CMS Collaboration [CMS Collaboration], CMS-PAS-HIG-13-035.

[52] G. Aad et al. [ATLAS Collaboration], Phys. Rev. Lett. 113, no. 17, 171801 (2014) arXiv:1407.6583 [hep-ex]].

[53] CMS Collaboration [CMS Collaboration], CMS-PAS-HIG-14-006.

[54] G. Aad et al. [ATLAS Collaboration], JHEP 1411, 056 (2014) arXiv:1409.6064 [hep-ex]].

[55] V. Khachatryan et al. [CMS Collaboration], JHEP 1410, 160 (2014) arXiv:1408.3316 [hep$\mathrm{ex}]]$.

[56] A. Arhrib, P. M. Ferreira and R. Santos, JHEP 1403, 053 (2014) arXiv:1311.1520 [hep-ph]].

[57] N. Craig, J. Galloway and S. Thomas, arXiv:1305.2424; A. Celis, V. Ilisie and A. Pich, JHEP 1312, 095 (2013); JHEP 1404, 128 (2014); P. S. B. Dev and A. Pilaftsis, JHEP 1412, 024 (2014).

[58] A. Barroso, P. M. Ferreira, I. P. Ivanov and R. Santos, JHEP 1306, 045 (2013) arXiv:1303.5098 [hep-ph]].

[59] J.-M. Gerard and M. Herquet, Phys. Rev. Lett. 98, 251802 (2007) hep-ph/0703051 [HEP$\mathrm{PH}]]$.

[60] J. Song and Y. W. Yoon, Phys. Rev. D 91, no. 11, 113012 (2015) arXiv:1412.5610 [hep-ph]].

[61] E. Barberio et al. [Heavy Flavor Averaging Group Collaboration], arXiv:0808.1297.

[62] M. Jung, A. Pich and P. Tuzon, JHEP 1011, 003 (2010).

[63] S. Schael et al. [ALEPH and DELPHI and L3 and OPAL and SLD and LEP Electroweak Working Group and SLD Electroweak Group and SLD Heavy Flavour Group Collaborations], Phys. Rept. 427, 257 (2006).

[64] J. Bernabeu, A. Pich and A. Santamaria, Nucl. Phys. B 363, 326 (1991); H. E. Haber and H. E. Logan, Phys. Rev. D 62, 015011 (2000); G. Degrassi and P. Slavich, Phys. Rev. D 81, 075001 (2010).

[65] M. Baak and R. Kogler, arXiv:1306.0571 [hep-ph]; H. Flacher, M. Goebel, J. Haller, A. Hocker, K. Monig and J. Stelzer, Eur. Phys. J. C 60, 543 (2009) [Erratum-ibid. C 71, 1718 (2011)].

[66] J. P. Lees et al. [BaBar Collaboration], Phys. Rev. Lett. 109, 101802 (2012) arXiv:1205.5442 [hep-ex]].

[67] R. Aaij et al. [LHCb Collaboration], Phys. Rev. Lett. 115, no. 11, 111803 (2015) 
arXiv:1506.08614 [hep-ex]].

[68] M. Huschle et al. [Belle Collaboration], arXiv:1507.03233 [hep-ex].

[69] A. Crivellin, C. Greub and A. Kokulu, Phys. Rev. D 86, 054014 (2012) arXiv:1206.2634 [hep-ph]].

[70] A. Crivellin, J. Heeck and P. Stoffer, arXiv:1507.07567 [hep-ph].

[71] G. Aad et al. [ATLAS Collaboration], Phys. Rev. D 90, no. 5, 052004 (2014) arXiv:1406.3827 [hep-ex]].

[72] V. Khachatryan et al. [CMS Collaboration], Eur. Phys. J. C 75, no. 5, 212 (2015) arXiv:1412.8662 [hep-ex]].

[73] G. Aad et al. [ATLAS and CMS Collaborations], Phys. Rev. Lett. 114, 191803 (2015) arXiv:1503.07589 [hep-ex]].

[74] CMS Collaboration [CMS Collaboration], CMS-PAS-HIG-13-026.

[75] P. Turner [CMS Collaboration], arXiv:1310.7859; B. A. Gonzalez, arXiv:1409.3340.

[76] S. Jung, J. Song and Y. W. Yoon, arXiv:1505.00291 [hep-ph].

[77] D. Dicus, A. Stange and S. Willenbrock, Phys. Lett. B 333, 126 (1994) hep-ph/9404359.

[78] A. Djouadi, L. Maiani, A. Polosa, J. Quevillon and V. Riquer, JHEP 1506, 168 (2015) arXiv:1502.05653 [hep-ph]].

[79] The ATLAS collaboration, ATLAS-CONF-2014-060, ATLAS-COM-CONF-2014-078.

[80] S. Chatrchyan et al. [CMS Collaboration], JHEP 1401, 096 (2014) arXiv:1312.1129 [hep-ex]].

[81] The ATLAS collaboration, ATLAS-CONF-2014-009, ATLAS-COM-CONF-2014-013.

[82] S. Chatrchyan et al. [CMS Collaboration], Phys. Rev. D 89, no. 9, 092007 (2014) arXiv:1312.5353 [hep-ex]].

[83] The ATLAS collaboration, ATLAS-CONF-2014-061, ATLAS-COM-CONF-2014-080.

[84] S. Chatrchyan et al. [CMS Collaboration], JHEP 1405, 104 (2014) arXiv:1401.5041 [hep-ex]].

[85] The ATLAS collaboration, ATLAS-CONF-2014-011, ATLAS-COM-CONF-2014-004.

[86] M. M. Llacer [ATLAS Collaboration], arXiv:1411.7638 [hep-ex].

[87] C. Vernieri [CMS Collaboration], arXiv:1411.4362 [hep-ex].

[88] G. Aad et al. [ATLAS Collaboration], JHEP 1501, 069 (2015) arXiv:1409.6212 [hep-ex]].

[89] S. Chatrchyan et al. [CMS Collaboration], Phys. Rev. D 89, no. 1, 012003 (2014) arXiv:1310.3687 [hep-ex]].

[90] N. Craig, F. D'Eramo, P. Draper, S. Thomas and H. Zhang, JHEP 1506, 137 (2015) 
arXiv:1504.04630 [hep-ph]].

[91] F. Larios, G. Tavares-Velasco and C. P. Yuan, Phys. Rev. D 66, 075006 (2002) hepph/0205204.

[92] D. L. Anderson, C. D. Carone and M. Sher, Phys. Rev. D 67, 115013 (2003) hep-ph/0303215].

[93] J. D. Clarke, R. Foot and R. R. Volkas, JHEP 1402, 123 (2014) arXiv:1310.8042 [hep-ph]].

[94] J. Baglio, O. Eberhardt, U. Nierste and M. Wiebusch, Phys. Rev. D 90, no. 1, 015008 (2014) arXiv:1403.1264 [hep-ph]].

[95] A. Djouadi, Phys. Rept. 459, 1 (2008) hep-ph/0503173.

[96] W. Kilian, M. Kramer and P. M. Zerwas, Phys. Lett. B 373, 135 (1996) hep-ph/9512355.

[97] A. Djouadi, W. Kilian, M. Muhlleitner and P. M. Zerwas, Eur. Phys. J. C 10, 27 (1999) hep-ph/9903229. 